\title{
MoHAmed LARBi ANNABI \\ Dépendance continue par rapport aux paramètres dans la bifurcation de Hopf-Takens de codimension 3
}

\author{
Annales de la faculté des sciences de Toulouse $5^{e}$ série, tome 12, \\ no 3 (1991), p. 295-327 \\ <http://www.numdam.org/item?id=AFST_1991_5_12_3_295_0>
}

(C) Université Paul Sabatier, 1991, tous droits réservés.

L'accès aux archives de la revue «Annales de la faculté des sciences de Toulouse » (http://picard.ups-tlse.fr/ annales/) implique l'accord avec les conditions générales d'utilisation (http://www.numdam.org/conditions). Toute utilisation commerciale ou impression systématique est constitutive d'une infraction pénale. Toute copie ou impression de ce fichier doit contenir la présente mention de copyright.

\section{NumDam}

Article numérisé dans le cadre du programme Numérisation de documents anciens mathématiques http://www.numdam.org/ 


\title{
Dépendance continue par rapport aux paramètres dans la bifurcation de Hopf-Takens de codimension 3
}

\author{
Mohamed Larbi ANNabi ${ }^{(1)}$
}

\begin{abstract}
RÉSUMÉ. - Le papier montre comment construire une $\left(C^{0}-C^{0}\right)$ équivalence, c'est-à-dire une $C^{0}$-équivalence dépendant continuement des paramètres, entre deux bifurcations génériques de Hopf-Takens de codimension 2 et 3 . La même étude est faite pour les familles génériques de champs de vecteurs à deux paramètres présentant des cycles triples.
\end{abstract}

\begin{abstract}
The paper shows how construct a $\left(C^{0}-C^{0}\right)$-equivalence, $C^{0}$-equivalence depending continuously on the parameters, between two generic Hopf-Takens bifurcations of codimention 2 and 3. It also includs the $\left(C^{0}-C^{0}\right)$ study of the generic 2-parameter unfolding of the triple cycle.
\end{abstract}

\section{Introduction}

Dans ce papier, je m'interresse à la relation entre l'équivalence faible ou la $\left(C^{0}\right.$-faible $\left.-C^{0}\right)$-équivalence, des germes de familles génériques de champs de vecteurs du plan, et l'équivalence forte ou la $\left(C^{0}-C^{0}\right)$-équivalence.

Rappelons d'abord les définitions suivantes.

- Deux telles familles $X_{\lambda}$ et $Y_{\lambda}$ sont $\left(C^{0}-\right.$ par fibre $\left.-C^{0}\right)$-équivalentes, s'il existe un homéomorphisme $\varphi$ de l'espace des paramètres tel que pour tout $\lambda$ il existe une $C^{0}$-équivalence $h_{\lambda}$ de $X_{\lambda}$ vers $Y_{\varphi(\lambda)}$, c'est-àdire que $h_{\lambda}$ est un homéomorphisme tel que $h_{\lambda}(0)=0$, envoyant les orbites de $X_{\lambda}$ sur celles de $Y_{\varphi(\lambda)}$ et préservant l'orientation.

- On dira que ces familles sont $\left(C^{0}-C^{0}\right)$-équivalentes si de plus l'application qui à $(\lambda, r)$ associe $h(\lambda, r)=h_{\lambda}(r)$ est de classe $C^{0}$.

(1) Département de Mathématique, Faculté des Sciences, 1060 Tunis, Tunisie 
On traitera le cas où la famille $X_{\lambda}$ est un déploiement générique de champs de vecteurs dans le plan $\mathbb{R}^{2}$ respectivement de Hopf en codimension 2, présentant un cycle triple et de Hopf en codimension 3.

Dans le cas d'une bifurcation de Hopf générique, les travaux de Takens [T] en décrivent un modèle $X_{\lambda}^{\text {mod }},\left(C^{\infty}-\right.$ faible $\left.-C^{\infty}\right)$-versel, cela veut dire que pour tout $\lambda$ il existe un difféomorphisme $h_{\lambda}$ tel que le champ $h_{\lambda} \star X_{\lambda}$ conjugué de $X_{\lambda}$ par $h_{\lambda}$ a les mêmes orbites périodiques et les mêmes singularités avec les mêmes types topologiques que le modèle $X_{\varphi(\lambda)}^{\text {mod }}$, où $\varphi$ envoie l'espace des paramètres de $X$ dans celui de $X^{\text {mod }}$; autrement dit, tel que la famille $\left\{h_{\lambda} \star X_{\lambda}\right\}_{\lambda}$ a exactement le même diagramme de bifurcation que le modèle avec les mêmes orbites périodiques, les mêmes singularités, etc., mais naturellement pas les mêmes orbites non périodiques. Nous revenons sur cette hypothèse par la suite. D'une façon précise, on a le théorème suivant de Takens $[\mathrm{T}]$.

ThÉonÈme .- Tout déploiement $X_{\lambda}$ d'une singularité de type $(k,-)$ est faiblement isomorphe $\dot{a}$ un déploiement induit de $X^{(k,-)}$.

On explique cet énoncé comme suit.

- $X_{\lambda}$ est un déploiement d'une singularité de type $(k,-)$ veut dire que $X_{0}$ est un champ générique dont les valeurs propres de la partie linéaire sont non nulles et se trouvent sur l'axe imaginaire. Cela veut aussi dire que le $(2 k+1)$-jet de $X_{0}$ à l'origine peut s'écrire à une $C^{\infty}$-équivalence près sous la forme :

$$
\left(x \frac{\partial}{\partial y}-y \frac{\partial}{\partial x}\right)-\left(x^{2}+y^{2}\right)^{k}\left(x \frac{\partial}{\partial x}+y \frac{\partial}{\partial y}\right) .
$$

- Le déploiement modèle $X^{(k,-)}$ est défini par :

$$
\begin{aligned}
X^{(k,-)}= & \left(x \frac{\partial}{\partial y}-y \frac{\partial}{\partial x}\right)-\left[\left(x^{2}+y^{2}\right)^{k}+\alpha_{1}\left(x^{2}+y^{2}\right)^{k-1}+\cdots\right. \\
& \left.\cdots+\alpha_{k-1}\left(x^{2}+y^{2}\right)+\alpha_{k}\right]\left(x \frac{\partial}{\partial x}+y \frac{\partial}{\partial y}\right) .
\end{aligned}
$$

- Pour les familles génériques à $k$ paramètres, la phrase faiblement isomorphe à un déploiement induit de $X^{k,-}$ signifie qu'à un $C^{\infty}$ changement des paramètres près les déploiements $X$ et $X^{k,-}$ ont exactement les mêmes diagrammes de bifurcation avec les mêmes orbites périodiques, les mêmes singularités, etc., mais pas nécessairement les mêmes orbites non périodiques. 
Rappelons, enfin, que la forme normale à une $C^{\infty}$-équivalence près d'un tel déploiement (voir $[\mathrm{T}]$ ) est la suivante :

$$
X=\left(x \frac{\partial}{\partial y}-y \frac{\partial}{\partial x}\right)-\left(g_{1}\left(\lambda ;\left(x^{2}+y^{2}\right)\right)+g_{2}(\lambda ;(x, y))\right)\left(x \frac{\partial}{\partial x}+y \frac{\partial}{\partial y}\right)
$$

$\left(\lambda \in \mathbb{R}^{k}\right)$ avec les propriétés suivantes.

i) $g_{2}$ est plate en tout point $(\lambda ; 0,0)$ et $g_{1}(0 ; 0,0)=0$;

ii) toute courbe intégrale fermée de $X$ est de la forme :

$$
\left\{(\lambda ; x, y), \lambda=\lambda_{1} ; x^{2}+y^{2}=R>0\right\},
$$

où $\lambda_{1} \in \mathbb{R}^{k}$ et $R>0$ sont fixés;

ii) le flot $D X: \mathbb{R}^{k} \times \mathbb{R}^{2} \times \mathbb{R} \rightarrow \mathbb{R}^{k} \times \mathbb{R}^{2}$ est globalement défini.

Pour résumer ces trois propriétés Takens [T] dit que $X$ vérifie la propriété de symétrie. La généricité en codimension $k$ se traduit par la propriété :

$$
g_{1}\left(0 ;\left(x^{2}+y^{2}\right)\right)=\left(x^{2}+y^{2}\right)^{k}+f_{1}\left(x^{2}+y^{2}\right) \text { avec } f_{1}(x)=o\left(x^{k}\right) .
$$

Dans ce papier, je montre qu'un déploiement générique de la forme (*) est $\left(C^{0}-C^{0}\right)$-équivalent à $X^{k,-}$ dans les cas $k=2$ et $k=3$. Il est à remarquer que dans $[R], R$. Roussarie a mentionné que cela ne peut pas être le cas pour les valeurs $k \geq 4$. Dans notre cas, les $\left(C^{0}-C^{0}\right)$-équivalences entre les champs s'obtiennent à partir de $\left(C^{0}-C^{0}\right)$-équivalences entre les champs s'obtiennent à partir de $\left(C^{0}-C^{0}\right)$-équivalence entre les difféomorphismes de Poincaré associés à ces champs. Or ces difféomorphismes sont de type selle-nœud transversalement à une certaine hypersurface $(S)$. La rigidité du problème est alors que la restriction d'une $\left(C^{0}-C^{0}\right)$-équivalence entre ces difféomorphismes à l'hypersurface $(S)$ est nécessairement une conjugaison entre les champs dans lesquels se plongent ces difféomorphismes.

Historiquement, cette rigidité a été découverte par [NPT], reprise par $[\mathrm{PR}]$ et considérée à nouveau par $[\mathrm{R}]$ qui mentionne que la $\left(C^{0}-\right.$ par fibre $C^{0}$ )-équivalence pour les bifurcations selles-nœud de difféomorphismes de la droite réelle peut donner lieu à des modules de $\left(C^{0}-C^{0}\right)$-équivalence. En particulier, il a été prouvé dans $[R]$ que pour de telles familles à trois paramètres et plus, il existe un module non dénombrable de familles génériques non $\left(C^{0}, C^{0}\right)$ équivalentes. Je trouve que ceci ne peut pas être le cas pour les familles à deux paramètres puisque dans ce cas la $\left(C^{0}-\right.$ par fibre $\left.-C^{0}\right)$-équivalence implique la $\left(C^{0}-C^{0}\right)$-équivalence (voir ci-après le cas du cycle triple). 
2. Un théorème de $\left(C^{0}-C^{0}\right)$-conjuguaison pour les difféomorphismes

Soient $m \in \mathbb{N}^{*}, E \subset \mathbb{R}^{m}, I$ un intervalle de $\mathbb{R}$ non vide et non réduit à un point. On note $\mathcal{F}(E, I, \mathbb{R})$ l'ensemble des triplets $(f, a, b)$ où $f: E \times I \rightarrow \mathbb{R}$ est une fonction de la classe $C^{\infty}, a: E \rightarrow I$ et $b: E \rightarrow I$ sont continues et telles que pour tout $\lambda \in E, a(\lambda)<b(\lambda)$. On notera $I(\lambda)=[a(\lambda), b(\lambda)]$.

\subsection{Définition de la propriété $(Y)$}

On dit qu'un triplet $(f, a, b) \in \mathcal{F}(E, I, \mathbb{R})$ vérifie la propriété $(Y)$ sur la partie $E_{1} \subset E$ si :

1) pour tout $\lambda \in E_{1}$, l'application qui à $r \in I(\lambda)$ associe $f_{\lambda}(r)=f(\lambda, r)$ est un difféomorphisme, préservant l'orientation de $I(\lambda)$ sur son image, de la classe $C^{\infty}$ en $(\lambda, r)$ ainsi que son inverse;

2) pour tout $\lambda \in E_{1}$, pour tout $r \in I(\lambda), f_{\lambda}(r) \geq r$, ou bien, pour tout $\lambda \in E_{1}$, pour tout $r \in I(\lambda), f_{\lambda}(r) \leq r$

3) pour tout $\lambda \in E_{1}, f_{\lambda}$ a au plus un point fixe dans $I(\lambda)$;

4) les intervalles $f_{\lambda}[I(\lambda)] \cap I(\lambda), f_{\lambda}\left(f_{\lambda}[I(\lambda)] \cap I(\lambda)\right) \cap I(\lambda)$ sont non vides et non réduits à un point.

\subsection{Plongement de difféomorphismes vérifiant $(Y)$ dans}

les champs $Y_{\lambda}$

Proposition 0. - Soit $(f, a, b) \in \mathcal{F}(E, I, \mathbb{R})$ vérifiant la propriété $(Y)$ sur une partie $E_{1} \subset E$, alors pour tout $\lambda \in E_{1}$, il existe un champ de vecteurs $Y_{\lambda}$ défini sur :

$$
J(\lambda)=I(\lambda) \cup f_{\lambda}(I(\lambda))
$$

tels que :

1) Si $Y_{\lambda, 1}$ est le flot au temps 1 du champ $Y_{\lambda}$ alors, on a la relation

$$
Y_{\lambda, 1}=f_{\lambda}
$$

2) si $\lambda_{n}$ est une suite de $E_{1}$, convergente vers $\lambda^{0} \in E_{1}$, alors pour $r_{n} \in J\left(\lambda_{n}\right)$ vérifiant $r_{n} \rightarrow r \in J\left(\lambda^{0}\right)$, on a $D^{i} Y_{\lambda_{n}}\left(r_{n}\right) \rightarrow D^{i} Y_{\lambda^{0}}(r)$ où 
Dépendance continue par rapport aux paramètres dans la bifurcation de Hopf-Takens

$D^{i}$ désigne la ième dérivée, et cela pour tout $i \geq 0$ si $r$ n'est pas une singularité de $Y_{\lambda^{0}}$ et pour tout $i=0,1$ si $r$ est une singularité de $Y_{\lambda^{0}}$.

Démonstration. - C'est une conséquence du théorème 2.5 de [Y].

Remarque 1. - La relation (A) implique que pour tout $r \in I(\lambda)$, on a :

$$
Y_{\lambda}\left(f_{\lambda}(r)\right)=f_{\lambda}^{\prime}(r) \cdot Y_{\lambda}(r)
$$

ce qui permet de prolonger $Y_{\lambda}$ d'une façon unique à la réunion suivante :

$$
\bigcup_{i \in\left\{j \in Z, f_{\lambda}^{j} \text { existe }\right\}} f_{\lambda}^{i}(I(\lambda))
$$

et telle que la relation (A) est toujours vérifiée.

\subsection{Propriété $\left(T_{Y}\right)$ pour les champs $Y_{\lambda}$}

Soient $E \subset \mathbb{R}^{m}, E^{\prime} \subset \mathbb{R}^{m-1}$ et $S: E^{\prime} \rightarrow \mathbb{R}$ continue. On notera $(S)$ le graphe de l'application $S$, c'est-à-dire :

$$
\begin{aligned}
(S)=\left\{\left(\lambda_{1}, \ldots, \lambda_{m-1}, \lambda_{m}\right) \in \mathbb{R}^{m}\right. & \mid\left(\lambda_{1}, \ldots, \lambda_{m-1}\right) \in E^{\prime} \\
& \text { et } \left.\lambda_{m}=S\left(\lambda_{1}, \ldots, \lambda_{m-1}\right)\right\},
\end{aligned}
$$

et on supposera que $(S) \subset E$. On notera $\left(S^{+}\right)$la région de $E$ au-dessus de $(S)$ :

$$
\begin{aligned}
\left(S^{+}\right)=\left\{\left(\lambda_{1}, \ldots, \lambda_{m-1}, \lambda_{m}\right) \in E \mid\right. & \left(\lambda_{1}, \ldots, \lambda_{m-1}\right) \in E^{\prime} \\
& \text { et } \left.\lambda_{m}>S\left(\lambda_{1}, \ldots, \lambda_{m-1}\right)\right\},
\end{aligned}
$$

et $\left(S^{-}\right)$la région de $E$ au-dessous de $(S)$ :

$$
\begin{aligned}
\left(S^{-}\right)=\left\{\left(\lambda_{1}, \ldots, \lambda_{m-1}, \lambda_{m}\right) \in E \mid\right. & \left(\lambda_{1}, \ldots, \lambda_{m-1}\right) \in E^{\prime} \\
& \text { et } \left.\lambda_{m}<S\left(\lambda_{1}, \ldots, \lambda_{m-1}\right)\right\} .
\end{aligned}
$$

Proposition 2. - Soit $(f, a, b) \in \mathcal{F}(E, I, \mathbb{R})$ vérifiant la propriété $(Y)$ sur la région $\left(S^{+}\right) \cup(S)$ avec $f_{\lambda}(r) \leq r$ (resp. $\left(S^{-}\right) \cup(S)$ avec $\left.f_{\lambda}(r) \geq r\right)$; on suppose de plus que:

1) pour tout $\lambda \in\left(S^{+}\right)$(resp. $\left(S^{-}\right)$), $f_{\lambda} n^{\prime}$ a pas de point fixe ;

2) pour tout $\lambda \in(S), f_{\lambda}$ a un unique point fixe $r_{\lambda} \in I(\lambda)$; 
3) pour tout $\lambda \in\left(S^{+}\right) \cup(S)$ (resp. $\left(S^{-}\right) \cup(S)$ ) et pour tout $r \in I(\lambda)$, on $a$ :

$$
\frac{\partial}{\partial \dot{\lambda}_{m}}\left[f_{\lambda}\right](r)<0 \text {. }
$$

Alors pour tout $\lambda \in(S)$, on $a$ :

$$
\frac{\partial}{\partial \lambda_{m}}\left[Y_{\lambda}\right]\left(r_{\lambda}\right)<0
$$

Démonstration. - Soit $\phi_{\lambda}(t, r)$ le flot de $Y_{\lambda}$. L'équation variationnelle s'écrit :

$$
\frac{\partial}{\partial t}\left[\frac{\partial}{\partial \lambda_{m}} \phi_{\lambda}\right](t, r)=D Y_{\lambda}\left(\phi_{\lambda}(t, r)\right)\left[\frac{\partial}{\partial \lambda_{m}} \phi_{\lambda}(t, r)\right]+\frac{\partial}{\partial \lambda_{m}}\left[Y_{\lambda}\right]\left(\phi_{\lambda}(t, r)\right) .
$$

Pour $\lambda \in(S)$ et $r=r_{\lambda}=\phi_{\lambda}\left(t, r_{\lambda}\right)$ pour $t \geq 0$, on a :

$$
\frac{\partial}{\partial t}\left[\frac{\partial}{\partial \lambda_{m}} \phi_{\lambda}\right]\left(t, r_{\lambda}\right)=D Y_{\lambda}\left(r_{\lambda}\right)\left[\frac{\partial}{\partial \lambda_{m}} \phi_{\lambda}\left(t, r_{\lambda}\right)\right]+\frac{\partial}{\partial \lambda_{m}}\left[Y_{\lambda}\right]\left(r_{\lambda}\right) .
$$

D'où, si l'on pose $A(\lambda)=D Y_{\lambda}\left(r_{\lambda}\right)$ alors :

$$
\begin{aligned}
\frac{\partial}{\partial \lambda_{m}} \phi_{\lambda}\left(t, r_{\lambda}\right) & =e^{t A(\lambda)} \frac{\partial}{\partial \lambda_{m}} \phi_{\lambda}\left(0, r_{\lambda}\right)+A(\lambda)^{-1}\left(e^{t A(\lambda)}-1\right) \frac{\partial}{\partial \lambda_{m}}\left[Y_{\lambda}\right]\left(r_{\lambda}\right) \\
& =A(\lambda)^{-1}\left(e^{t A(\lambda)}-1\right) \frac{\partial}{\partial \lambda_{m}}\left[Y_{\lambda}\right]\left(r_{\lambda}\right),
\end{aligned}
$$

car $\partial / \partial \lambda_{m} \phi_{\lambda}\left(0, r_{\lambda}\right)=0$ puisque $\phi_{\lambda}(0, r)=r$ ne dépend pas de $\lambda_{m}$. D'où le résultat en faisant $t=1$.

Remarque 3. - Cette proposition implique que pour tout $\lambda^{0} \in(S)$, il existe $\epsilon>0$, il existe $\eta>0$ tel que si $\lambda \in E$ et $r \in I(\lambda)$ vérifient $\left|\lambda-\lambda^{0}\right|<\epsilon$ et $\left|\boldsymbol{r}-r_{\lambda^{0}}\right|<\eta$ alors :

$$
\frac{\partial}{\partial \lambda_{m}}\left[Y_{\lambda}\right]\left(r_{\lambda}\right)<0
$$

Proposition 4. - Si en plus des hypothèses de la proposition 2, les fonctions $a$ et $b$ admettent des dérivées partielles par rapport $a ̀ \lambda_{m}$ et

$$
\frac{\partial b}{\partial \lambda_{m}}(\lambda) \leq 0, \quad \frac{\partial a}{\partial \lambda_{m}}(\lambda) \geq 0,
$$


Dépendance continue par rapport aux paramètres dans la bifurcation de Hopf-Takens

$\lambda \in\left(S^{+}\right) \cup(S)$ (resp. $\left(S^{-}\right) \cup(S)$ ), alors pour tout $\lambda^{0} \in(S)$, il existe $\epsilon=\epsilon\left(\lambda^{0}\right)>0$ tel que la fonction $\lambda \rightarrow t_{(a, b)}(\lambda)$ représentant le temps mis pour aller de $b(\lambda)$ à $a(\lambda)$ par le champ $Y_{\lambda}$, c'est-à-dire celle qui à $\lambda \in\left(\varsigma^{+}\right)$ (resp. $\left(S^{-}\right)$) associe

$$
t_{(a, b)}(\lambda)=\int_{b(\lambda)}^{a(\lambda)} \frac{\mathrm{d} r}{Y(\lambda, r)}
$$

vérifie pour $\left|\lambda-\lambda^{0}\right|<\epsilon$ l'inégalité $(\mathrm{t})$ suivante :

$$
\frac{\partial}{\partial \lambda_{m}}\left[t_{(a, b)}\right](\lambda)<0, \quad(\text { resp. }>0) .
$$

Démonstration. - Nous développons la démonstration dans le cas où la propriété $(Y)$ est vérifiée dans la région $\left(S^{+}\right) \cup(S)$ avec $f_{\lambda}(r) \leq r$. L'autre cas est similaire.

Soit $\epsilon_{1}>0$ et $\eta>0$ tels que si $\lambda \in E$ et $r \in I(\lambda)$ vérifient $\left|\lambda-\lambda^{0}\right|<\epsilon_{1}$ et $\left|r-r_{\lambda^{0}}\right|<\eta$ et $Y_{\lambda}(r)$ existe, alors :

$$
\frac{\partial}{\partial \lambda_{m}}\left[Y_{\lambda}\right](r)<0
$$

Soient $n_{0}, n_{1}$ des entiers tels que $r^{0}=f^{-n_{0}}\left(\lambda^{0}, a\left(\lambda^{0}\right)\right)$ et $r^{1}=$ $f^{n_{1}}\left(\lambda^{0}, b\left(\lambda^{0}\right)\right)$ vérifient $\left|r^{0}-r_{\lambda^{0}}\right|<\eta$ et $\left|r^{1}-r_{\lambda^{0}}\right|<\eta$. La continuité de $f$ implique qu'il existe $\epsilon>0, \epsilon_{1}>\epsilon$ tels que si $\lambda \in E,\left|\lambda-\lambda^{0}\right|<\epsilon$, alors $r^{0}(\lambda)=f^{-n_{0}}(\lambda, a(\lambda))$ et $r^{1}(\lambda)=f^{n_{1}}(\lambda, b(\lambda))$ vérifient encore $\left|r^{0}(\lambda)-r_{\lambda^{0}}\right|<\eta$ et $\left|r^{1}(\lambda)-r_{\lambda^{0}}\right|<\eta$. Or, on a :

$$
\begin{aligned}
t_{(a, b)}(\lambda) & =\int_{b(\lambda)}^{a(\lambda)} \frac{\mathrm{d} r}{Y(\lambda, r)} \\
& =\int_{b(\lambda)}^{r^{1}(\lambda)} \frac{\mathrm{d} r}{Y(\lambda, r)}+\int_{r^{1}(\lambda)}^{r^{0}(\lambda)} \frac{\mathrm{d} r}{Y(\lambda, r)}+\int_{r^{0}(\lambda)}^{a(\lambda)} \frac{\mathrm{d} r}{Y(\lambda, r)} \\
& =n_{0}+n_{1}+\int_{r^{1}(\lambda)}^{r^{0}(\lambda)} \frac{\mathrm{d} r}{Y(\lambda, r)}
\end{aligned}
$$

D'où :

$$
\begin{aligned}
& \frac{\partial}{\partial \lambda_{m}}\left[t_{(a, b)}\right](\lambda)= \frac{\left[\partial r^{0} / \partial \lambda_{m}(\lambda)\right]}{Y\left(\lambda, r^{0}(\lambda)\right)}-\frac{\left[\partial r^{1} / \partial \lambda_{m}(\lambda)\right]}{Y\left(\lambda, r^{1}(\lambda)\right)}+ \\
&-\int_{r^{1}(\lambda)}^{r^{0}(\lambda)}\left[\frac{\partial Y}{\partial \lambda_{m}}(\lambda, r)\right] \frac{\mathrm{d} r}{[Y(\lambda, r)]^{2}}<0 . \\
&-301-
\end{aligned}
$$




\section{Remarques 5}

1) Cette proposition implique que l'inégalité $(t)$ est vérifiée en particulier pour tout paramètre $\lambda$ compris entre la surface $(S)$ et une surface $\left(S^{1}\right)$ vérifiant pour tout $\left(\lambda_{1}, \ldots, \lambda_{m-1}\right) \in E^{\prime}$ :

$$
\begin{aligned}
& S\left(\lambda_{1}, \ldots, \lambda_{m-1}\right)<S^{1}\left(\lambda_{1}, \ldots, \lambda_{m-1}\right) \leq S\left(\lambda_{1}, \ldots, \lambda_{m-1}\right)+\epsilon(\lambda), \\
& \quad \text { où } \lambda=\left(\lambda_{1}, \ldots, \lambda_{m-1}, S\left(\lambda_{1}, \ldots, \lambda_{m-1}\right)\right) .
\end{aligned}
$$

2) Elle nous permet (voir la démonstration du théorème 6 ci-après), en paramétrant par le temps $t_{(a, b)}(\lambda)$ le segment :

$$
] S\left(\lambda_{1}^{0}, \ldots, \lambda_{m-1}^{0}\right), S^{1}\left(\lambda_{1}^{0}, \ldots, \lambda_{m-1}^{0}\right)\right]
$$

où $\left(\lambda_{1}^{0}, \ldots, \lambda_{m-1}^{0}\right)$ est fixé dans $E^{\prime}$, de construire (voir [NPT] ou [R]) une $\left(C^{0}-C^{0}\right)$-conjugaison entre deux telles familles $\left\{Y_{\lambda}\right\}$ et $\left\{Y_{\lambda}^{\prime}\right\}$ associées à des difféomorphismes $\left\{f_{\lambda}\right\}$ et $\left\{g_{\lambda}\right\}$ et par suite d'obtenir une $\left(C^{0}-C^{0}\right)$-conjugaison des $\left\{f_{\lambda}\right\}$ vers les $\left\{g_{\lambda}\right\}$. D'une façon précise, on a :

THÉORÈme 6. - Soient $(f, a, b) \in \mathcal{F}(E, I, \mathbb{R})$ et $(g, c, d) \in \mathcal{F}\left(E, I^{\prime}, \mathbb{R}\right)$ deux triplets vérifiant la propriété $(Y)$ sur la même région $\left(S^{+}\right) \cup(S)$ avec $f_{\lambda}(r) \leq r$ et $g_{\lambda}(r) \leq r$ (resp. $\left(S^{-}\right) \cup(S)$ avec $f_{\lambda}(r) \geq r$ et $\left.g_{\lambda}(r) \geq r\right)$. Si chacun de ces deux triplets vérifie les hypothèses de la proposition 2, et si d'une part $\partial b / \partial \lambda_{m}(\lambda)$ et $\partial d / \partial \lambda_{m}(\lambda)$ existent et sont négatifs ou nuls et d'autre part $\partial a / \partial \lambda_{m}(\lambda)$ et $\partial c / \partial \lambda_{m}(\lambda)$ existent et sont positifs ou nuls, alors les difféomorphismes $\left\{f_{\lambda}\right\}$ et $\left\{g_{\lambda}\right\}$ sont $\left(C^{0}-C^{0}\right)$-conjugués au voisinage de $(S)$ dans $\left[\left(S^{+}\right) \cup(S)\right]$. D'une façon précise il existe deux surfaces $\left(S^{2}\right)$ et $\left(S^{\prime 2}\right)$ au-dessus de $(S)$ telles que si $V$ désigne la région comprise entre (S) et $\left(S^{2}\right)$ alors il existe :

$$
h:(\lambda, r) \in V \times I \rightarrow h(\lambda, r) \in I^{\prime}
$$

continue telle que pour tout $\lambda$ l'application $r \rightarrow h_{\lambda}(r)=h(\lambda, r)$ est une $C^{0}$-conjuguaison de $f_{\lambda}$ vers $g_{\varphi(\lambda)}$, où $\varphi$ est un homéomorphisme de $V$ dans la région comprise entre $\left(S^{2}\right)$ et $\left(S^{2}\right)$ laissant $(S)$ invariant, envoyant $\left(S^{2}\right)$ sur $\left(S^{2}\right)$ et vérifiant pour tout $\lambda=\left(\lambda_{1}, \ldots, \lambda_{m-1}, \lambda_{m}\right)$ :

$$
\varphi\left(\lambda_{1}, \ldots, \lambda_{m-1}, \lambda_{m}\right)=\left(\lambda_{1}, \ldots, \lambda_{m-1}, \varphi_{m}(\lambda)\right) .
$$

Démonstration. - Nous développons la démonstration dans le cas où la propriété $(Y)$ est vérifiée dans la région $\left(S^{+}\right) \cup(S)$ avec $f_{\lambda}(r) \leq r$. L'autre cas est similaire. 
Soit $Y(\lambda, r)$ (resp. $\left.Y^{\prime}(\lambda, r)\right)$ le champ associé à $(f, a, b)$ (resp. $\left.(g, c, d)\right)$ par la proposition 0 et soit $S^{1}$ (resp. $S^{11}$ ) une hypersurface continue au-dessus de $(S)$ telle que :

$$
\frac{\partial}{\partial \lambda_{m}}\left[t_{(a, b)}\right](\lambda)<0, \quad\left(\text { resp. } \frac{\partial}{\partial \lambda_{m}}\left[t_{(c, d)}\right](\lambda)<0\right)
$$

pour tout $\lambda$ compris entre $(S)$ et $\left(S^{1}\right)$ (resp. $(S)$ et $\left(S^{1}\right)$ ).

Considérons deux hypersurfaces $\left(S^{2}\right)$ et $\left(S^{2}\right)$ de $E$ comprises entre $(S)$ et $\left(S^{1}\right)$ (resp. $(S)$ et $\left.\left(S^{1}\right)\right)$ telles que pour tout $\lambda=\left(\lambda_{1}, \ldots, \lambda_{m-1}, \lambda_{m}\right) \in$ $\left(S^{2}\right)$ et $\lambda^{\prime}=\left(\lambda_{1}, \ldots, \lambda_{m-1}, \lambda_{m}^{\prime}\right) \in\left(S^{2}\right)$, on a

$$
t_{(a, b)}(\lambda)=t_{(c, d)}\left(\lambda^{\prime}\right)
$$

posons pour $\lambda=\left(\lambda_{1}, \ldots, \lambda_{m-1}, \lambda_{m}\right)$ compris entre $(S)$ et $\left(S^{2}\right)$,

$$
\varphi(\lambda)=\left(\lambda_{1}, \ldots, \lambda_{m-1}, \lambda_{m}^{\prime}\right)=\lambda^{\prime}
$$

$\lambda^{\prime}$ étant défini d'une façon unique par la relation $(*)$.

La conjugaison annoncée est alors définie pour $\lambda$ compris entre $(S)$ et $\left(S^{2}\right)$ par :

$$
\begin{cases}h\left(\lambda, Y_{\lambda, t}(a(\lambda))\right)=\left(\varphi(\lambda), Y_{\varphi(\lambda), t}^{\prime}(c(\lambda))\right) & \text { si } t \leq 0 \\ h\left(\lambda, Y_{\lambda, t}(b(\lambda))\right)=\left(\varphi(\lambda), Y_{\varphi(\lambda), t}^{\prime}(d(\lambda))\right) & \text { si } t \geq 0 \\ h\left(\lambda, r_{\lambda}\right)=\left(\varphi(\lambda), r_{\varphi(\lambda)}^{\prime}\right) & \text { si } \lambda \in(S)\end{cases}
$$

où $Y_{\lambda, t}$ (resp. $\left.Y_{\varphi(\lambda), t}^{\prime}\right)$ est le flot de $Y_{\lambda}\left(\right.$ resp. $\left.Y_{\varphi(\lambda)}^{\prime}\right)$.

\subsection{Deux lemmes de prolongement}

On désinge par $\Lambda_{1}$ un fermé d'un espace métrique $(\Lambda, d)$ admettant un voisinage $V\left(\Lambda_{1}\right)$ qui se retracte continuement sur $\Lambda_{1}$, c'est-à-dire qu'il existe une application continue $\rho: V\left(\Lambda_{1}\right) \rightarrow \Lambda_{1}$ vérifiant $\rho(\lambda)=\lambda$ pour tout $\lambda \in \Lambda_{1}$. On suppose de plus qu'il existe $r>0$ tel que :

$$
V\left(\Lambda_{1}\right) \supset\left\{x \in \Lambda, d\left(x, \Lambda_{1}\right)<r\right\} .
$$

On désignera aussi par $J$ un intervalle de $\mathbb{R}$ et on notera $I=[0,1]$. 
LEMME 1. - Toute application $h$ continue de $\Lambda_{1} \times I$ à valeurs dans $I$ telle que pour tout $\lambda \in \Lambda_{1}, h_{\lambda}: I \rightarrow I$ définie par $h_{\lambda}(x)=h(\lambda, x)$ est un homéomorphisme vérifiant $h(\lambda, 0)=0$ et $h(\lambda, 1)=1$ se prolonge en une application continue $H$ :

$$
H: \Lambda \times I \rightarrow I
$$

telle que les $H_{\lambda},(\lambda \in \Lambda)$, sont des homéomorphismes vérifiant $H(\lambda, 0)=0$ et $H(\lambda, 1)=1$.

Démonstration. - L'application de $H: I \times \Lambda \rightarrow I$ suivante répond à la question :

$$
\begin{cases}H(\lambda, x)=x+\left[1-\frac{\inf \left(1, d\left(\lambda, \Lambda_{1}\right)\right)}{r}\right] \cdot[h(\rho(\lambda), x)-x] & \text { si } \lambda \in V\left(\Lambda_{1}\right) \\ H(\lambda, x)=x & \text { si } \lambda \notin V\left(\Lambda_{1}\right) .\end{cases}
$$

Les applications $H_{\lambda}(x)=H(\lambda, x)$ sont des homéomorphismes parce que si une application $x \rightarrow x+\varphi(x)$ est un homéomorphisme qui préserve l'orientation alors, pour tout $\tau \in[0,1]$, l'application $x \rightarrow x+\tau \varphi(x)$ est aussi un homéomorphisme.

LeMme 2. - Soient $\left(f_{\lambda}\right)_{\lambda \in \Lambda}$ et $\left(g_{\lambda}\right)_{\lambda \in \Lambda}$ deux familles de difféomorphismes sur les intervalles $I$ et $J$ (dont les extrémités peuvent dépendre continuement de $\lambda$ ) de $\mathbb{R}$, topologiquement triviales et de même type topologique; alors toute $\left(C^{0}-\mathrm{Id}\right)$-conjuguaison $\varphi(\lambda, \cdot), \lambda \in \Lambda_{1}$ de $\left(f_{\lambda}\right)_{\lambda \in \Lambda_{1}}$ vers $\left(g_{\lambda}\right)_{\lambda \in \Lambda_{1}}$ telle que $\varphi(\lambda, a(\lambda))=(\lambda, c(\lambda))$ s'étend en une $\left(C^{0}-\mathrm{Id}\right)$ conjuguaison, $\phi(\lambda, \cdot)$, de $\left(f_{\lambda}\right)_{\lambda \in \Lambda}$ vers $\left(g_{\lambda}\right)_{\lambda \in \Lambda}$.

On entend par topologiquement triviale une famille $\left(f_{\lambda}\right)_{\lambda \in \Lambda}$ telle que si $\lambda_{0} \in \Lambda$ alors il existe, pour tout $\lambda \in \Lambda$, une conjuguaison $\varphi(\lambda, \cdot)$ dépendant continuement de $\lambda$ de $f_{\lambda}$ vers $f_{\lambda_{0}}$.

Deux familles topologiquement triviales sont de même type topologique si un élément de l'une est conjugué à un élément de l'autre.

Démonstration. - Vue le caractère unidimentionel, la conjuguaison en dehors des points fixes est décrite sur des domaines fondamentaux et sur chaque paire de domaines fondamentaux $I_{\lambda}=[a(\lambda), b(\lambda)]$ et $J_{\lambda}=$ $[c(\lambda), d(\lambda)]$ qui se correspondent, on fait la démonstration suivante.

Considérons l'application continue $h: \Lambda_{1} \times[0,1] \rightarrow \Lambda_{1} \times[0,1]$ rendant 
Dépendance continue par rapport aux paramètres dans la bifurcation de Hopf-Takens

le diagramme suivant commutatif :

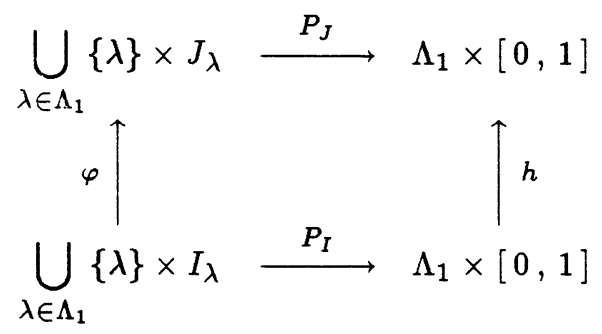

où $P_{I}(\lambda,(1-t) a(\lambda)+t b(\lambda))=(\lambda, t)$ pour $t \in[0,1]$ et $P_{J}$ est défini d'une façon analogue.

Soit $H: \Lambda \times[0,1] \rightarrow \Lambda \times[0,1]$ le prolongement de $h$ donné par le lemme 1. Cette application $H$ permet de prolonger $\varphi$ en une application continue $\phi$ :

$$
\phi: \bigcup_{\lambda \in \Lambda}\{\lambda\} \times I_{\lambda} \rightarrow \bigcup_{\lambda \in \Lambda}\{\lambda\} \times J_{\lambda}
$$

La définition finale de $\phi: \Lambda \times I \rightarrow \Lambda \times J$ s'obtient par saturation, c'està-dire à partir de la relation :

$$
\phi\left(f_{\lambda}(x), \lambda\right)=\left(g_{\lambda}(\phi(x, \lambda)), \lambda\right)
$$

Les problèmes de frontières peuvent être résolus par un choix convenable des domaines fondamentaux.

\section{Le cas Hopf en codimension 2}

\subsection{Position du problème}

Soit $X$ un déploiement versel de la classe $C^{\infty}$ de la singularité de codimension 2 :

$X_{(0,0)}=\left(x \frac{\partial}{\partial y}-y \frac{\partial}{\partial x}\right)-\left[\left(x^{2}+y^{2}\right)^{2}+f_{1}\left(x^{2}+y^{2}\right)+f_{2}(x, y)\right]\left(x \frac{\partial}{\partial x}+y \frac{\partial}{\partial y}\right)$

avec $f_{1}(z)=o\left(z^{2}\right)$ et $f_{2}(x, y)$ plate en $(0,0)$. 


\section{L. Annabi}

D'après $[\mathrm{T}]$, on peut supposer qu'à une $C^{\infty}$-équivalence près, $X$ s'écrit :

$$
\begin{aligned}
X= & \left(x \frac{\partial}{\partial y}-y \frac{\partial}{\partial x}\right)-\left(g_{1}\left(\alpha, \beta ;\left(x^{2}+y^{2}\right)\right)+\right. \\
& \left.+g_{2}(\alpha, \beta ;(x, y))\right)\left(x \frac{\partial}{\partial x}+y \frac{\partial}{\partial y}\right)
\end{aligned}
$$

avec :

i) $g_{2}$ est plate en tout point $(\alpha, \beta ; 0,0)$ et $g_{1}(0,0 ; 0)=0$;

ii) toute courbe intégrale fermée de $X$ est de la forme :

$$
\left\{(\alpha, \beta ; x, y),(\alpha, \beta)=\left(\alpha_{1}, \beta_{1}\right) ; x^{2}+y^{2}=R>0\right\}
$$

où $\left(\alpha_{1}, \beta_{1}\right) \in \mathbb{R}^{2}$ et $R>0$ sont fixés;

ii) le flot $D X: \mathbb{R}^{2} \times \mathbb{R}^{2} \times \mathbb{R} \rightarrow \mathbb{R}^{2} \times \mathbb{R}^{2}$ est globalement défini.

Takens a démontré dans [T] que $X$ est faiblement isomorphe à un déploiement induit du déploiement $X^{2,-}$ suivant :

$$
X^{2,-}=\left(x \frac{\partial}{\partial y}-y \frac{\partial}{\partial x}\right)-\left(\left(x^{2}+y^{2}\right)^{2}+\alpha\left(x^{2}+y^{2}\right)+\beta\right)\left(x \frac{\partial}{\partial x}+y \frac{\partial}{\partial y}\right)
$$

qui s'écrit en coordonnées polaires :

$$
X^{2,-}=\frac{\partial}{\partial \theta}-\left(r^{4}+\alpha r^{2}+\beta\right) r \frac{\partial}{\partial r}
$$

On cherchera à démontrer que le déploiement $X$ est $\left(C^{0}-C^{0}\right)$-équivalent à $X^{2,-}$.

\subsection{Les diagrammes de bifurcations et les portraits de phases}

3.2.1. Diagramme de bifurcation et portraits de phases de $X^{2,-}$

Rappelons [T] que le diagramme de bifurcation et les portraits de phases de $X^{2,-}$ sont décrits par la figure 1 . 


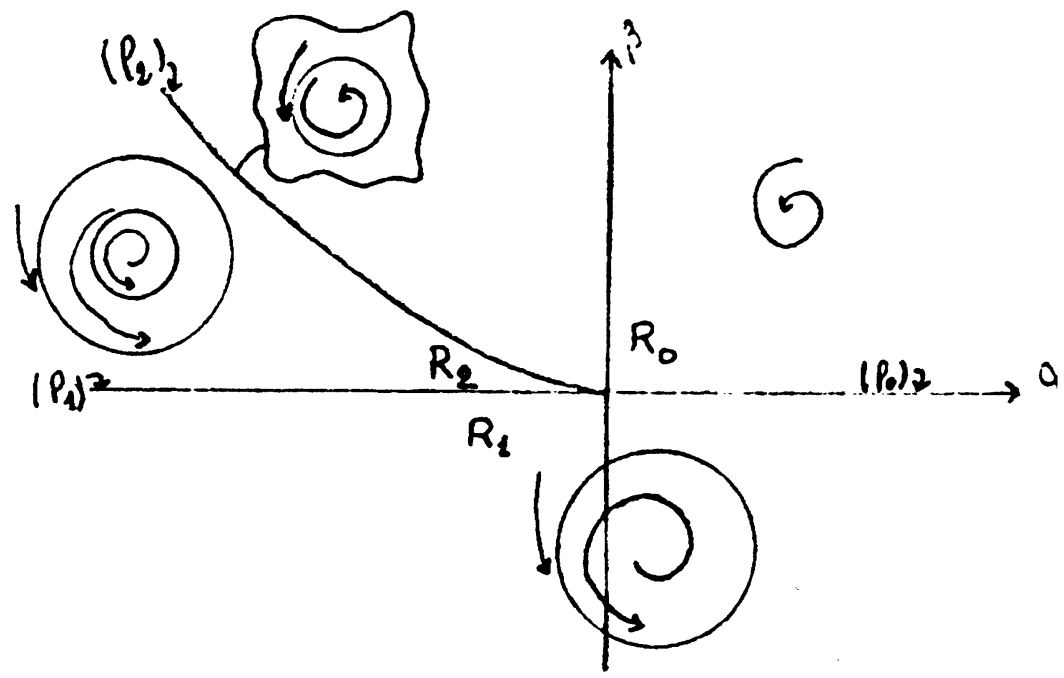

Fig. 1

Pour $(\alpha, \beta)$ appartenant à la ligne $\left(\ell_{2}\right)$ définie par la demi-parabole $\beta=\alpha^{2} / 4$ située dans le demi-plan $\alpha<0$, le champ $X_{(\alpha, \beta)}^{2,-}$ admet une seule orbite fermée, attractive à l'extérieur et répulsive à l'intérieur.

On a aussi une seule orbite fermée pour :

- $(\alpha, \beta)$ appartenant à la région $R_{1}$ définie par $\beta<0$,

- $(\alpha, \beta)$ appartenant à la ligne $\left(\ell_{1}\right)$ définie $\operatorname{par} \beta=0$ et $\alpha<0$.

Dans chacun de ces deux cas l'unique orbite fermée est attractive.

Lorsque $(\alpha, \beta)$ appartient à la région $R_{2}$ située dans le demi-plan $\alpha<0$ et limitée par les lignes $\left(\ell_{1}\right)$ et $\left(\ell_{2}\right)$, le champ $X_{(\alpha, \beta)}^{2,-}$ admet deux orbites fermées, qui sont deux cercles centrés à l'origine. Le cercle intérieur étant répulsif et l'autre attractif.

Le champ $X_{(\alpha, \beta)}^{2,-}$ n'admet pas d'orbite fermée lorsque :

- $(\alpha, \beta)$ appartient à la ligne $\left(\ell_{0}\right)$ définie $\operatorname{par} \beta=0$ et $\alpha>0$,

- $(\alpha, \beta)$ appartient à la région $R_{0}$ du plan des paramètres.

On désignera par $r_{1}(\alpha, \beta) \geq r_{2}(\alpha, \beta)$ les rayons des orbites fermées de $X_{(\alpha, \beta)}^{2,-}$ lorsqu'elles existent, $r_{3}(\alpha, \beta)=\left(r_{1}(\alpha, \beta)+r_{2}(\alpha, \beta)\right) / 2$ avec la convention que $r_{2}(\alpha, \beta)=0$ si $(\alpha, \beta) \in R_{1} \cup\left(\ell_{1}\right)$ et $r_{4}(\alpha, \beta)=r_{2}(\alpha, \beta) / 2$. Il est à noter que pour toute valeur du paramètre $(\alpha, \beta)$, l'origine $(0,0) \mathrm{du}$ 


\section{L. Annabi}

plan des phases est une singularité du champ $X_{(\alpha, \beta)}^{2,-}$. Cette singularité est un foyer répulsif lorsque $(\alpha, \beta) \in R_{1} \cup\left(\ell_{1}\right)$, il est attractif sinon.

Les lignes $\left(\ell_{i}\right)_{i=0,1,2}$ sont donc des lignes de bifurcations de $X^{2,-}$.

\subsubsection{Diagramme de bifurcation et portraits de phases de $X$}

D'après la généricité de $X$, on a les deux conséquences suivantes.

Première conséquence

La généricité de $X$ veut dire que si :

$$
g_{1}\left(\alpha, \beta ; r^{2}\right)=B(\alpha, \beta)+A(\alpha, \beta) r^{2}+O\left(r^{4}\right)
$$

alors l'application qui ̀̀ $(\alpha, \beta)$ associe $(A(\alpha, \beta), B(\alpha, \beta))$ est de rang maximum au point $(0,0)$, c'est-à-dire que c'est un difféomorphisme local. On notera $\xi$ sa réciproque et en notant $g_{1}$ la fonction qui à $\left(\alpha, \beta ; r^{2}\right)$ associe $g_{1}\left(\xi(\alpha, \beta) ; r^{2}\right)=\beta+\alpha r^{2}+O\left(r^{4}\right)$ et $X$ le déploiement qui à $(\alpha, \beta, \theta, r)$ associe $X(\xi(\alpha, \beta), \theta, r)$ alors, on a :

$$
X((\alpha, \beta), \theta, r)=\frac{\partial}{\partial \theta}-G_{1}(\alpha, \beta, \theta, r) \frac{r \partial}{\partial r}
$$

avec

$$
G_{1}(\alpha, \beta, \theta, r)=-\left(g_{1}\left(\alpha, \beta ; r^{2}\right)+g_{2}(\alpha, \beta ;(r \cos \theta, r \sin \theta))\right)
$$

on a en particulier

$$
\frac{\partial G_{1}}{\partial \beta}(0,0, \theta, 0)=-1 .
$$

La continuité de $\partial G_{1} / \partial \beta$ permet de conclure que $\partial G_{1} / \partial \beta(\alpha, \beta, \theta, r)<0$ pour $(\alpha, \beta, \theta, r)$ voisin de $(0,0, \theta, 0)$, c'est-à-dire que si $G(\alpha, \beta, \theta, r)=$ $r G_{1}(\alpha, \beta, \theta, r)$ alors, on a l'inégalité $\left(T_{X}\right)$ suivante :

$$
\frac{\partial G}{\partial \beta}(\alpha, \beta, \theta, r)<0
$$

pour $(\alpha, \beta, r)$ voisin de $(0,0,0)$ et $\theta \in[0,2 \pi], r \neq 0$.

Deuxième conséquence

D'après $[\mathrm{T}]$, il existe un $C^{\infty}$-morphisme faible $\phi$ de la forme :

$$
\begin{gathered}
\phi(\mu, x, y)=\left(\varphi(\mu), \Lambda\left(\mu, x^{2}+y^{2}\right)(x, y)\right) \\
-308-
\end{gathered}
$$


où $\mu=(\alpha, \beta)$ est le paramètre de $X$ vers $X^{2,-}$, avec la propriété que $\Lambda>0$ sur un voisinage de l'origine. La généricité de $X$ implique que $\varphi$ est difféomorphisme local. Ainsi, la substitution de $\Phi \star X$ à $X$ nous permet de supposer sans restreindre la généralité que $X$ et $X^{2,-}$ ont le même diagramme de bifurcation, les mêmes orbites périodiques, les mêmes singularités sur chaque fibre avec les mêmes types topologiques; cela veut dire que $X$ et $X^{2,-}$ ont les mêmes portraits de phases. Pour résumer ces propriétés, on dira que $X$ et $X^{2,-}$ sont faiblement identiques au voisinage de l'origine. Naturellement $X$ et $X^{2,-}$ n'ont pas nécessairement les mêmes orbites non périodiques. De plus, la forme $(*)$ et la propriété $\left(T_{X}\right)$ ne sont pas altérées par cette substitution.

Soit alors $U=U_{1} \times U_{2}$ un voisage de l'origine de $\mathbb{R}^{2} \times \mathbb{R}^{2}$ tel que les restrictions de $X$ et $X^{2,-}$ à $U$ soient faiblement identiques.

Soient, en outre, $r_{0}>0, \beta_{0}>0$ et $\alpha_{0}=\beta_{0}^{1 / 2}$ tels que le compact $C$ :

$$
C=\left\{(\alpha, \beta),-\alpha_{0} \leq \alpha \leq \alpha_{0} \text { et }-\beta_{0} \leq \beta \leq \beta_{0}\right\}
$$

vérifie $C \subset U_{1}$ et tel que $r_{0}$ est un réel vérifiant, d'une part :

$$
r_{0}>\sup \left\{r_{1}(\alpha, \beta),(\alpha, \beta) \in C \cap\left(\overline{R_{1}} \cup \overline{R_{2}}\right)\right\}
$$

et d'autre part, le disque de l'espace des phases de centre l'origine et de rayon $r_{0}$ soit inclus dans $U_{2}$.

On supposera de plus que l'inégalité $\left(T_{X}\right)$ est vérifiée pour tout $(\alpha, \beta) \in C$ et $\left.r \in] 0, r_{0}\right]$.

\subsubsection{Propriété rotationnelle}

Fixons $\alpha \in\left[-\alpha_{0}, 0[\right.$ et $\left.r \in] 0, r_{0}\right]$ et considérons l'application $\tau$ qui à $\beta$, tel que $(\alpha, \beta, \theta, r) \in U$, pour $\theta \in[0,2 \pi]$, associe l'angle $\tau(\beta)$ de la tangente à l'orbite $D X\left(\alpha, \beta, \theta_{0}, r\right)$, du champ $X_{(\alpha, \beta)}$, au point $\left(\alpha, \beta, \theta_{0}, r\right)$ de l'axe polaire $\theta=\theta_{0}$, avec ce même axe polaire $\theta=\theta_{0}$. On a :

$$
\operatorname{tg}(\tau(\beta))=\frac{r}{\dot{r}}=\frac{r}{G\left(\alpha, \beta, \theta_{0}, r\right)} ;
$$

donc $\tau$ est une fonction croissante de $\beta$ lorsque $(\alpha, \beta) \in C$ et $\left.r \in] 0, r_{0}\right]$ (fig. 2). 


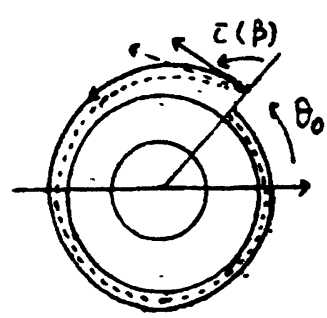

$\beta<P_{2}(\alpha)$

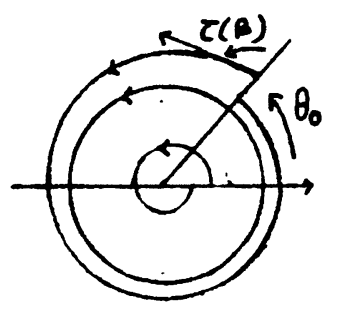

$\beta=l_{2}(\alpha)$

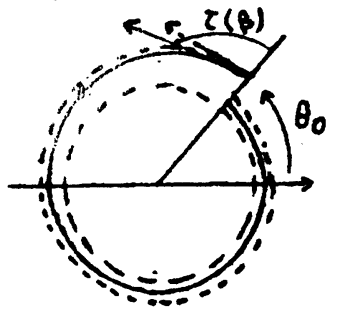

$\beta<\rho_{2}(\alpha)$

Fig. 2

\subsection{4. Évolution des points fixes sur l'axe des $r$}

Le dessin suivant (fig. 3) décrit l'évolution de l'axe des $r$ lorsque $(\alpha, \beta)$ varie dans $U_{1}$ (resp. $(\alpha, \beta)$ parcourant une ligne fermée entourant $(0.0)$ ).
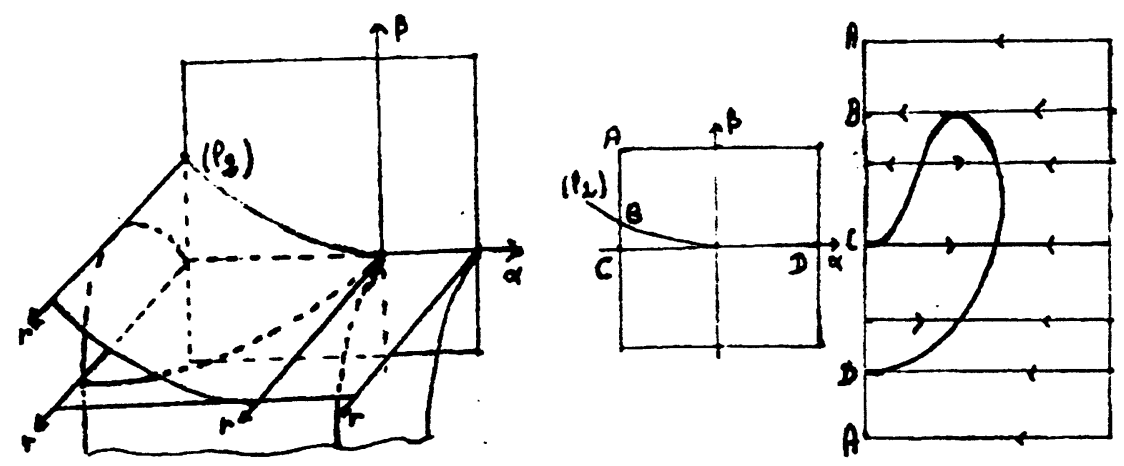

Fig. 3

3.3. Conjugaison continue entre les difféomorphismes de Poincaré associé à $X$ et à $X^{2,-}$

Soient $\mathcal{P}$ et $\mathcal{P}^{2,-}$ les applications de Poincaré associées respectivement à $X$ et $X^{2,-}$ relativement à la demi-droite des $x>0$. 
Dépendance continue par rapport aux paramètres dans la bifurcation de Hopf-Takens

Notons :

$$
\begin{cases}r_{4}^{\prime}(\alpha, \beta)=\inf \left\{r_{4}\left(\alpha, \ell_{2}(\alpha)\right), \frac{\mathcal{P}_{(\alpha, \beta)}^{2}\left(r_{0}\right)}{2}\right\} & \text { si } \alpha \leq 0 \\ r_{4}^{\prime}(\alpha)=0 & \text { si } \alpha \geq 0\end{cases}
$$

On adopte une définition analogue pour $r_{4}(\alpha, \beta)$ relativement au champ $X^{2,-}$.

\subsubsection{Conjuguaison au-dessus de $\left(\ell_{2}\right)$}

On considère $E=\left\{(\alpha, \beta) \in C \mid \alpha \leq 0\right.$ et $\left.\beta \geq \ell_{2}(\alpha)\right\}$. On pose $f(\lambda, r)=$ $\mathcal{P}(\lambda, r)$ et $g(\lambda, r)=\mathcal{P}^{2,-}(\lambda, r)$, où $\lambda \in E$ et $r \in I=\left[0, r_{0}\right]$. Enfin soient $a(\lambda)=r_{4}^{\prime}(\lambda), b(\lambda)=r_{0}, c(\lambda)=r_{4}(\lambda), d(\lambda)=r_{0}$ et $(S)=\left(\ell_{2}\right)$.

La propriété rotationelle implique que les triplets $(f, a, b)$ et $(g, c, d)$ ainsi définis vérifient les hypothèses du théorème 6 sur $[E \backslash\{(0,0)\}] \times I$. Il existe donc deux lignes $(\ell)$ et $\left(\ell^{\prime}\right)$ continues, décroissantes, incluses dans $E$ et vérifiant $\ell(0)=\ell^{\prime}(0)=0$. Il existe aussi une $\left(C^{0}-C^{0}\right)$-conjugaison de la restriction de $\mathcal{P}$ à $V \times I$ vers la restriction de $\mathcal{P}^{2,-}$ à $V^{\prime} \times I$ où $V$ (resp. $V^{\prime}$ ) est la région de $E \backslash\{(0,0)\}$ comprise entre $\left(\ell_{2}\right)$ et $(\ell)$ (resp. entre $\left(\ell_{2}\right)$ et $\left.\left(\ell^{\prime}\right)\right)$.

Un prolongement par continuité permet d'avoir le résultat suivant.

LEMME .- $\Pi$ existe une $\left(C^{0}-C^{0}\right)$-conjugaison de $\mathcal{P}$ vers $\mathcal{P}^{2,-}$ qui à $(\lambda, r) \in[V \cup\{(0,0)\}] \times I$ associe $(\varphi(\lambda), h(\lambda, r)) \in E \times I$, vérifiant en particulier :

$$
h\left(\lambda, r_{0}\right)=r_{0} \quad \text { et } \quad h\left(\lambda, r_{4}^{\prime}(\lambda)\right)=r_{4}(\varphi(\lambda))
$$

$\varphi$ étant un homéomorphisme de $V \cup\{(0,0)\}$ dans $V^{\prime} \cup\{(0,0)\}$ de la forme

$$
\begin{cases}\varphi(\alpha, \beta)=\left(\alpha, \varphi_{2}(\alpha, \beta)\right) & \text { si } \beta \neq \ell_{2}(\alpha) \\ \varphi(\alpha, \beta)=(\alpha, \beta) & \text { si } \beta=\ell_{2}(\alpha)\end{cases}
$$

\subsubsection{Prolongement de la conjuguaison à $C \times\left[0, r_{0}\right]$}

Finalement on considère un homéomorphisme $A$ de $C$ respectant le diagramme de bifurcation et coïncidant avec $\varphi$ sur la région $V \cup\{(0,0)\}$. Il est bien entendu que les familles :

$$
f(\lambda, r)=\mathcal{P}(\lambda, r) \quad \text { et } \quad g(\lambda, r)=\mathcal{P}^{2,-}(A(\lambda), r)
$$


ne sont pas topologiquement triviales mais leurs restrictions à la région de l'espace des phases définies par $\left.] r_{2}(\lambda), r_{0}\right]$ (resp. $\left.\left.\left.] r_{1}(\lambda), r_{2}\right],\right] 0, r_{1}(\lambda)\right]$ ) sont topologiquement triviales et de même type topologique (fig. 4).
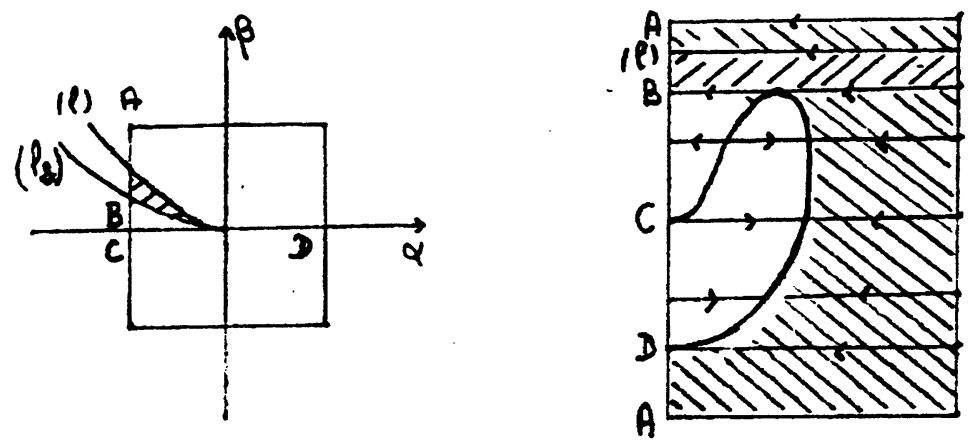

Fig. 4

Le lemme 2 de prolongement du paragraphe 2.4 s'applique donc et permet de prolonger $(\varphi, h)$ à $C \times\left[0, r_{0}\right]$ en une $\left(C^{0}-C^{0}\right)$-conjugaison de $\mathcal{P}$ vers $\mathcal{P}^{2,-}$.

3.4. $\left(C^{0}-C^{0}\right)$-conjugaison de la forme normale de $X$ vers $X^{2,-}$

C'est l'application définie au voisinage de $((0,0) ;(0,0))$ qui au point $M=((\alpha, \beta) ;(\theta, r))$ du niveau $(\alpha, \beta)$ d'argument $\theta$ et de rayon $r$ dans le plan des phases de $X_{(\alpha, \beta)}$ associe le point :

$$
H(M)=\left(\varphi(\alpha, \beta) ;\left(\theta, P_{\theta}^{-}\left(h\left((\alpha, \beta), P^{+}(M)\right)\right)\right)\right)
$$

dans laquelle nous avons :

- $P^{+}(M)$ est la première intersection de la demi-orbite positive de $M$ par le champ $X_{(\alpha, \beta)}$ avec le demi-axe des $x>0$;

- $P_{\theta}^{-}$est l'application qui à un point $N$ du demi-axe des $x>0$ associe la première intersection de sa demi-orbite négative par le champ $X_{\varphi(\alpha, \beta)}^{2,-}$ avec la demi-droite d'argument $\theta$. 
Dépendance continue par rapport aux paramètres dans la bifurcation de Hopf-Takens

\section{Le cas du cycle triple}

\subsection{Position du problème}

Soit $X$ une famille verselle de champs de vecteurs de la classe $C^{\infty}$ sur $\mathbb{R}^{2}$ à deux paramètres ayant une fonction de déplacement $f_{(\alpha, \beta)}(r)=$ $\mathcal{P}_{(\alpha, \beta)}(r)-r, \mathcal{P}$ étant l'application de Poincaré au voisinage d'un cycle triple. On suppose que :

$$
f_{(\alpha, \beta)}(r)=f(\alpha, \beta, r)=-\left[Q(\alpha, \beta)+P(\alpha, \beta) r+R(\alpha, \beta) r^{2}+r^{3}+o\left(r^{3}\right)\right],
$$

avec $Q(0,0)=P(0,0)=R(0,0)=0$.

Nous montrons, dans ce paragraphe, que la famille $\mathcal{P}_{\lambda}=r+f_{\lambda}$ est $\left(C^{0}-C^{0}\right)$-conjuguée à la famille $\mathcal{Q}_{\lambda}=r+g_{\lambda}$, où on a :

$$
g_{(\alpha, \beta)}(r)=g(\alpha, \beta, r)=-\left[\beta+\alpha r+r^{3}\right] .
$$

\subsection{Diagramme de bifurcation}

Le théorème de préparation implique qu'il existe des fonctions $C^{\infty}: h_{1}$, $a, b$ et $c$ telles que

$$
f(\lambda, r)=-h_{1}(\lambda, r)\left(c(\lambda)+b(\lambda) r+a(\lambda) r^{2}+r^{3}\right)
$$

avec $h_{1}(0,0)>0$ et $\lambda=(\alpha, \beta)$.

La versalité veut dire que l'application qui à $\lambda=(\alpha, \beta)$ associe $(b(\lambda), c(\lambda))$ est de rang maximum au point $(0,0)$. Par la suite, en écrivant :

$$
f(\lambda, r)=-h_{1}\left(\lambda, r+\frac{a(\lambda)}{3}\right)\left(B(\lambda)+A(\lambda)\left(r+\frac{a(\lambda)}{3}\right)+\left(r+\frac{a(\lambda)}{3}\right)^{3}\right) .
$$

L'application qui à $\lambda=(\alpha, \beta)$ associe $\varphi(\lambda)=(A(\lambda), B(\lambda))$ est aussi de rang maximum au point $(0,0)$, donc $\varphi$ est un $C^{\infty}$-difféomorphisme au voisinage de $(0,0)$. Notons :

$$
\Lambda_{\lambda}(r)=\Lambda(\lambda, r)=r+\frac{a(\lambda)}{3} \quad \text { et } \quad \phi(\lambda, r)=(\Lambda(\lambda, r), \varphi(\lambda)) .
$$


Il est clair que $\phi$ est un difféomorphisme vérifiant :

$$
f_{\lambda}(r)=f(\lambda, r)=h(\phi(\lambda, r)) g(\phi(\lambda, r)) .
$$

Be plus, on a :

$$
\phi^{-1}(\lambda, r)=\left(\left(\Lambda_{\varphi^{-1}(\lambda)}\right)^{-1}(r), \varphi^{-1}(\lambda)\right)
$$

d'où :

$$
f\left(\phi^{-1}(\lambda, r)\right)=h(\lambda, r) g(\lambda, r),
$$

c'est-à-dire que pour tout $\lambda$, on a :

$$
\Lambda_{\varphi^{-1}(\lambda)} \circ \mathcal{P}_{\varphi^{-1}(\lambda)} \circ\left(\Lambda_{\varphi^{-1}(\lambda)}\right)^{-1}=r+h(\lambda, r) g(\lambda, r) .
$$

Ainsi donc à une $\left(C^{\infty}-C^{\infty}\right)$-conjuguaison près on peut supposer que :

$$
f(\lambda, r)=h(\lambda, r) g(\lambda, r),
$$

et que les familles $\mathcal{P}_{\lambda}$ et $\mathcal{Q}_{\lambda}$ ont exactement le même diagramme de bifurcation, les mêmes points fixes sur chaque fibre avec les mêmes types topologiques. Pour résumer ces propriétés, on dira que ces familles sont faiblement identiques au voisinage de l'origine.

Le diagramme de bifurcation est ainsi donné par :

$$
\beta+\alpha r+r^{3}=0, \quad \alpha+3 r^{2}=0,
$$

ou encore

$$
\beta=2 r^{3}, \quad \alpha=-3 r^{2} .
$$

Il s'agit d'un “ cusp " dont l'allure est donnée par la figure 5.

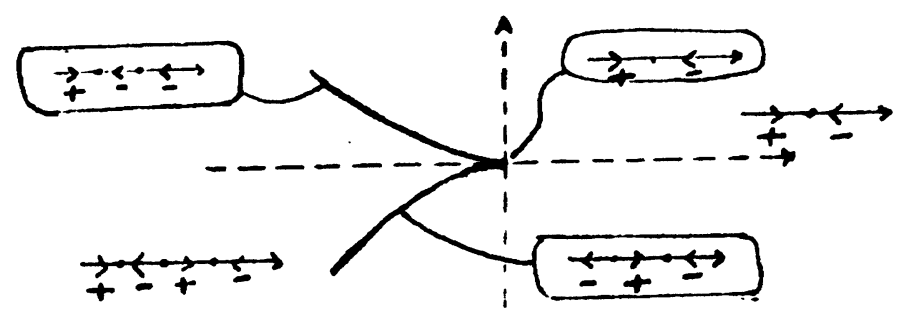

Fig. 5 
Nous avons donc, pour l'équation $\beta+\alpha r+r^{3}=0$ :

- une racine réelle unique lorsque $\lambda$ est dans la région $R_{1}$;

- trois racines réelles distinctes lorsque $\lambda$ est dans la région $R_{3}$;

- deux racines réelles : $r_{1}$ simple et $r_{2}=-r_{1} / 2$ double, donc :

$$
\begin{cases}r_{1}>r_{2} & \text { si } \lambda \in S_{2,-} \\ r_{2}>r_{1} & \text { si } \lambda \in S_{2,+}\end{cases}
$$

- une racine réelle triple si $\alpha=\beta=0$.

D'où le dessin suivant (fig. 6) décrivant l'évolution des zéros de $f_{\lambda}$ (et par suite des points fixes de $\mathcal{P}_{\lambda}$ ) lorsque $\lambda$ varie au voisinage du point triple
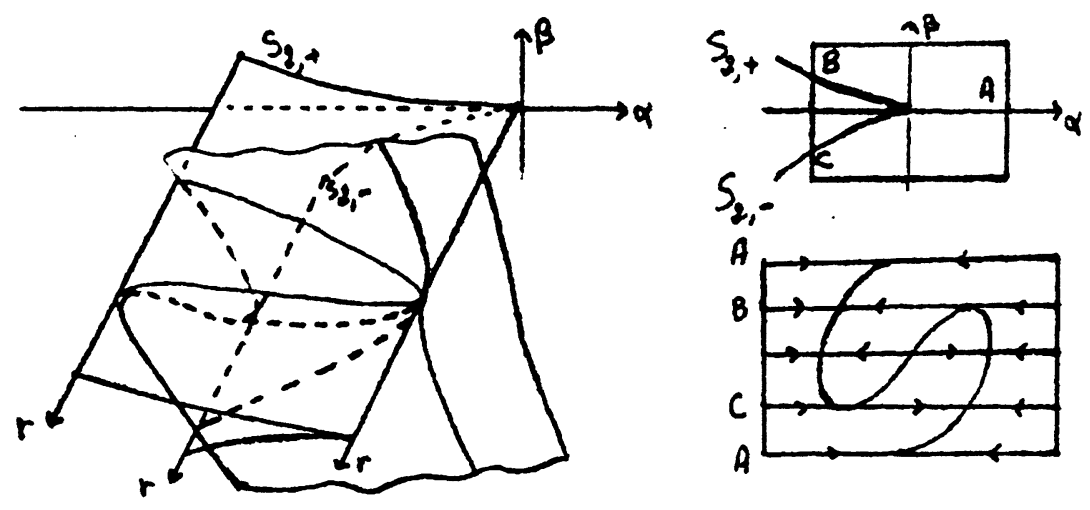

Fig. 6

\subsection{La conjuguaison}

Soit $U=U_{1} \times U_{2}$ un voisinage de l'origine de $\mathbb{R}^{2} \times \mathbb{R}$ sur lequel les familles $\mathcal{P}_{\lambda}$ et $\mathcal{Q}_{\lambda}$ sont faiblement identiques.

Soient $r_{0}>0, \alpha_{0}>0$ et $\beta_{0}>2\left(\alpha_{0} / 3\right)^{3 / 2}$ tels que le compact :

$$
C=\left\{(\alpha, \beta) ;-\alpha_{0} \leq \alpha \leq \alpha_{0} \text { et }-\beta_{0} \leq \beta \leq \beta_{0}\right\}
$$

vérifie $C \subset U_{1}$ et tel que $I=\left[-r_{0}, r_{0}\right] \subset U_{2}$ et $r_{0}>\sup \left\{|r|, g_{\lambda}(r)=0\right.$ et $\lambda \in C\}$.

Comme dans le cas Hopf en codimension 2, le théorème 6 implique l'existence d'une $\left(C^{0}-C^{0}\right)$-conjuguaison, qui à $(\lambda, r)$ associe $(\varphi(\lambda), h(\lambda, r))$ 
des difféomorphismes $\left\{\mathcal{P}_{\lambda}\right\}$ vers les difféomorphismes $\left\{\mathcal{Q}_{\lambda}\right\}$, définie pour $(\lambda, r) \in C \times I$ tel que :

- ou bien $\lambda$ est au-dessus de $S_{2,+} \cup\{(0,0)\}$ (i.e. $\lambda=(\alpha, \beta) ; \beta \geq S_{2,+}(\alpha)$ ou $\alpha=0$ et $\beta=0)$ et $r \in\left[r_{1}(\lambda), r_{0}\right]$;

- ou bien $\lambda$ est au-dessous de $S_{2,-} \cup\{(0,0)\}$ (i.e. $\lambda=(\alpha, \beta) ; \beta \leq S_{2,-}(\alpha)$ ou $\alpha=0$ et $\beta=0)$ et $r \in\left[-r_{0}, r_{1}(\lambda)\right]$.

Le prolongement continu de cette $\left(C^{0}-C^{0}\right)$-conjuguaison se fait à l'aide du lemme 2 de prolongement (paragraphe 2.4) de façon similaire à celle du cas Hopf en codimension 2.

\subsection{L'équivalence entre les champs}

Avec une construction usuelle, en utilisant le rapport des temps par exemple, on peut étendre la conjuguaison des applications de Poincaré en une équivalence entre les champs.

\section{Le cas Hopf en codimension 3}

\subsection{Position du problème}

Soit $X$ un déploiement versel de la classe $C^{\infty}$ de la singularité de codimension 3 :

$$
X_{(0,0)}=\left(x \frac{\partial}{\partial y}-y \frac{\partial}{\partial x}\right)-\left[\left(x^{2}+y^{2}\right)^{3}+f_{1}\left(x^{2}+y^{2}\right)+f_{2}(x, y)\right]\left(x \frac{\partial}{\partial x}+y \frac{\partial}{\partial y}\right)
$$

avec

$$
f_{1}(z)=o\left(z^{3}\right) \text { et } f_{2}(x, y) \text { plate en }(0,0)
$$

D'après $[\mathrm{T}]$, on peut supposer à une $C^{\infty}$-équivalence près que $X$ s'écrit :

$$
\begin{aligned}
X= & \left(x \frac{\partial}{\partial y}-y \frac{\partial}{\partial x}\right)-\left(g_{1}\left((\alpha, \beta, \gamma) ;\left(x^{2}+y^{2}\right)\right)+\right. \\
& \left.+g_{2}((\alpha, \beta, \gamma) ;(x, y))\right)\left(x \frac{\partial}{\partial x}+y \frac{\partial}{\partial y}\right)
\end{aligned}
$$

avec :

i) $g_{2}$ est plate en tout point $((\alpha, \beta, \gamma) ;(0,0))$ et $g_{1}((0,0,0) ; 0)=0$; 
Dépendance continue par rapport aux paramètres dans la bifurcation de Hopf-Takens

ii) toute courbe intégrale fermée de $X$ est de la forme :

$$
\left\{\left((\alpha, \beta, \gamma) ;\left(x^{\prime}, y^{\prime}\right)\right),(\alpha, \beta, \gamma)=\left(\alpha_{1}, \beta_{1}, \gamma_{1}\right) ; x^{2}+y^{2}=R>0\right\}
$$

où $\left(\alpha_{1}, \beta_{1}, \gamma_{1}\right) \in \mathbb{R}^{3}$ et $R>0$ sont fixés;

iii) le flot $D X: \mathbb{R}^{3} \times \mathbb{R}^{2} \times \mathbb{R} \rightarrow \mathbb{R}^{3} \times \mathbb{R}^{2}$ est globalement défini.

Takens dans [T] a montré que $X$ est faiblement isomorphe à un déploiement induit du déploiement $X^{3,-}$ suivant :

$$
\begin{aligned}
X^{3,-}= & \left(x \frac{\partial}{\partial y}-y \frac{\partial}{\partial x}\right)-\left(\left(x^{2}+y^{2}\right)^{3}+\alpha\left(x^{2}+y^{2}\right)^{2}+\right. \\
& \left.+\beta\left(x^{2}+y^{2}\right)+\gamma\right)\left(x \frac{\partial}{\partial x}+y \frac{\partial}{\partial y}\right)
\end{aligned}
$$

qui s'écrit en coordonnées polaires :

$$
X^{3,-}=\frac{\partial}{\partial \theta}-\left(r^{6}+\alpha r^{4}+\beta r^{2}+\gamma\right) \frac{r \partial}{\partial r} .
$$

On cherchera à démontrer que le déploiement $X$ est $\left(C^{0}-C^{0}\right)$-équivalent à $X^{3,-}$.

\subsection{Les diagrammes de bifurcations et les portraits de phases}

5.2.1. Diagramme de bifurcation et portraits de phases de $X^{3,-}$

Rappelons ([T]) que le diagramme de bifurcation (fig. 7) de $X^{3,-}$ est la réunion du plan $\{\gamma=0\}$ et de la surface $(S)$ paramétrée par :

$(S):$

$$
\beta=-3 a^{2}-2 a \alpha, \quad \gamma=2 a^{3}+\alpha a^{2}, \quad a \geq 0 .
$$


M. L. Annabi

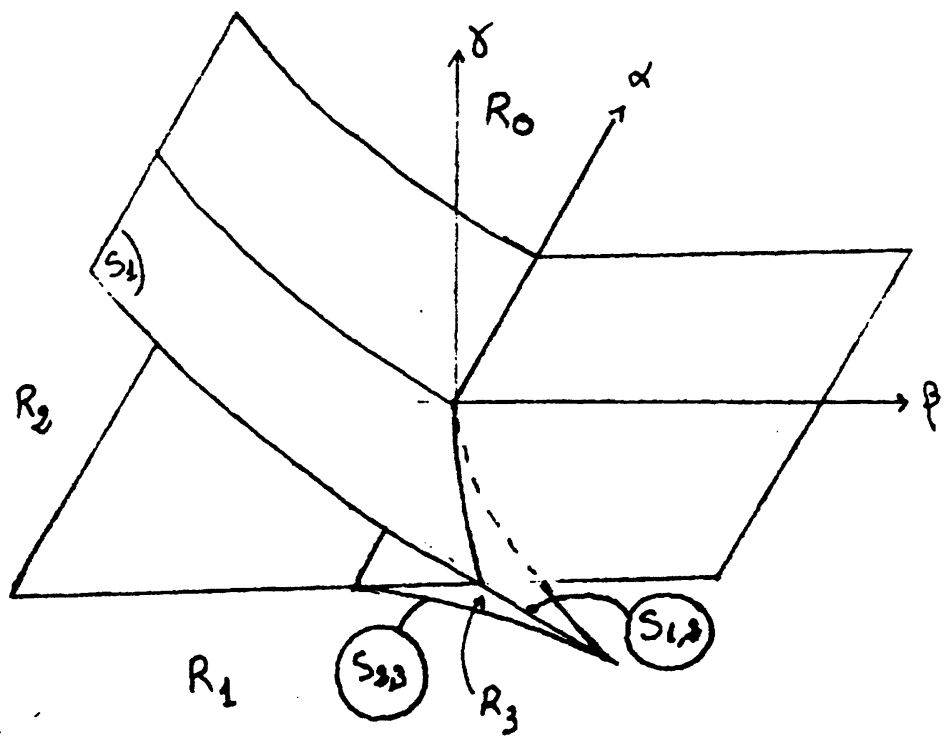

Fig. 7

Les portraits de phases relatifs aux régions $R_{0}, R_{1}, R_{2}$ et $R_{3}$ sont décrits par la figure 8.

$R_{0}$ :

$R_{2}$ :

\section{$\sigma$}

$R_{1}$ :

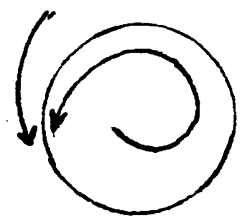

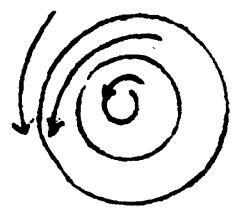

$R_{3}:$

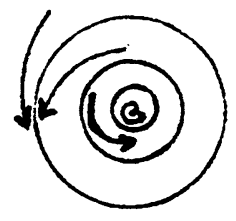

Fig. 8 
Dépendance continue par rapport aux paramètres dans la bifurcation de Hopf-Takens

Les portraits de phases associés à un paramètre $(\alpha, \beta, \gamma) \in\{\gamma=0\} \cup(S)$ sont décrits par les figures 9,10 , et 11 .

$\alpha>0$ :

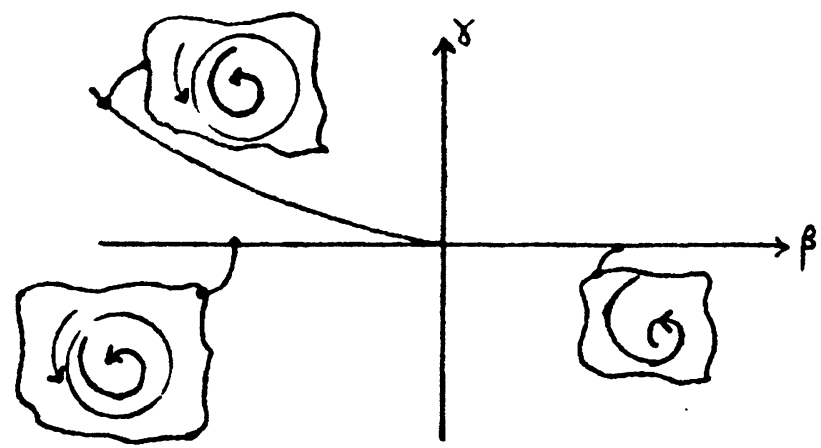

Fig. 9

$\alpha<0$ :

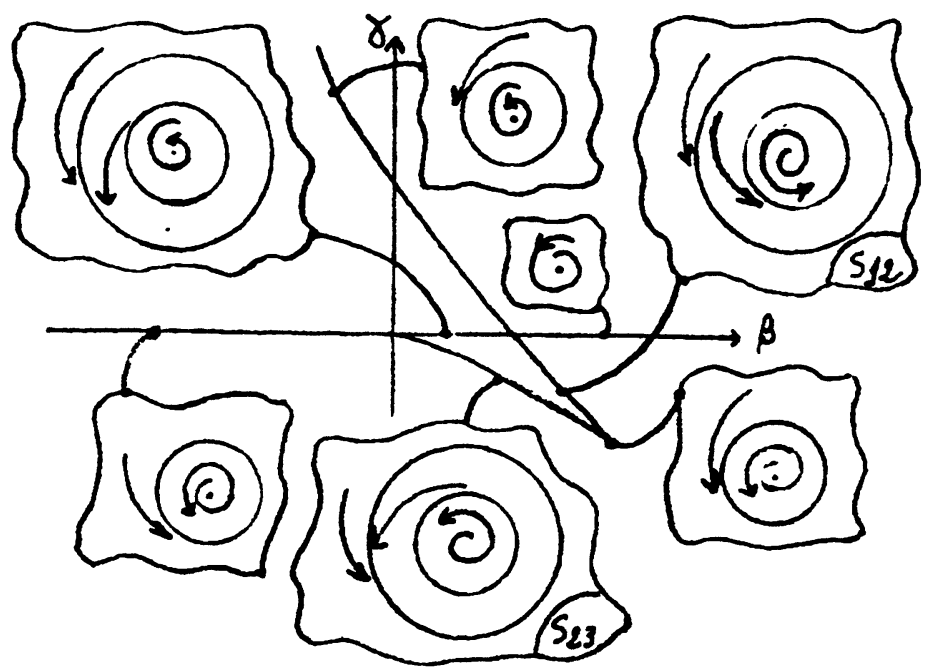

Fig. 10 
$\alpha=0$ :

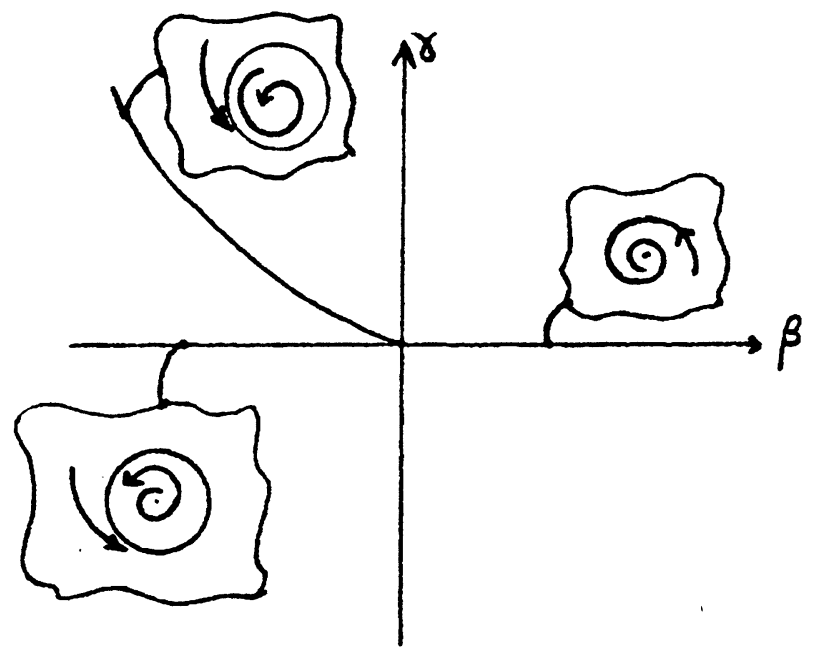

Fig. 11

Ainsi, on a la description suivante des orbites du champ $X_{(\alpha, \beta, \gamma)}^{3,-}$ au voisinage de l'origine du plan des phases.

- Il n'y a pas de cycle limite, lorsque $(\alpha, \beta, \gamma)$ est dans la région $R_{0}$ ou bien lorsque $(\alpha, \beta, \gamma)$ est dans la partie du plan $\{\gamma=0\}$ séparant les régions $R_{1}$ et $R_{0}$; c'est aussi le cas si $\alpha=\beta=\gamma=0$. L'origine étant une singularité attractive.

- Il y a un cycle limite attractif, lorsque $(\alpha, \beta, \gamma)$ est dans la région $R_{1}$ ou bien lorsque $(\alpha, \beta, \gamma)$ est dans la partie du plan $\{\gamma=0\}$ séparant les régions $R_{1}$ et $R_{2}$. C'est un cercle centré à l'origine, son rayon sera noté $r_{1}(\alpha, \beta, \gamma)$. - Il y a un cycle limite attractif de l'extérieur, répulsif de l'intérieur, lorsque $(\alpha, \beta, \gamma)$ est dans la partie $\left(S_{1}\right)$ de la surface $(S)$ définie par :

$\left(S_{1}\right): \quad \beta=-3 a^{2}-2 a \alpha, \quad \gamma=2 a^{3}+\alpha a^{2}, \quad a>-\frac{\alpha}{2} \geq 0$,

ou bien lorsque $(\alpha, \beta, \gamma)$ est sur la ligne $\left(\ell_{2}\right)$ de $(S)$ définie par

$\left(\ell_{2}\right)$ :

$\alpha=-2 a, \quad \beta=a^{2}, \quad \gamma=0, \quad a \geq 0$.

C'est un cercle centré à l'origine, son rayon sera noté $r_{1,2}(\alpha, \beta, \gamma)$. On a en particulier $r_{1,2}(\alpha, \beta, \gamma)=a$. 
- Il y a deux cycles limites qui sont des cercles centrés à l'origine, l'un attractif de rayon $r_{1}(\alpha, \beta, \gamma)$ et l'autre est répulsif de rayon $r_{2}(\alpha, \beta, \gamma)<$ $r_{1}(\alpha, \beta, \gamma)$, lorsque $(\alpha, \beta, \gamma)$ est dans la région $R_{2}$ ou bien lorsque $(\alpha, \beta, \gamma)$ est dans la partie du plan $\{\gamma=0\}$ séparant les régions $R_{2}$ et $R_{3}$. Remarquons que d'une part dans ce cas l'origine est une singularité stable, et que d'autre part, si $\left(\alpha_{0}, \beta_{0}, \gamma_{0}\right)$ est dans $\left(S_{1}\right)$ alors :

$$
\begin{aligned}
\lim _{\substack{(\alpha, \beta, \gamma) \rightarrow\left(\alpha_{0}, \beta_{0}, \gamma_{0}\right) \\
(\alpha, \beta, \gamma) \in R_{2}}} r_{1}(\alpha, \beta, \gamma) & =\lim _{\substack{(\alpha, \beta, \gamma) \rightarrow\left(\alpha_{0}, \beta_{0}, \gamma_{0}\right) \\
(\alpha, \beta, \gamma) \in R_{2}}} r_{2}(\alpha, \beta, \gamma) \\
& =r_{1,2}\left(\alpha_{0}, \beta_{0}, \gamma_{0}\right) .
\end{aligned}
$$

- Deux cycles limites, qui sont des cercles centrés à l'origine, apparaissent également dans chacun des deux cas suivant.

i) $(\alpha, \beta, \gamma)$ est dans la partie $\left(S_{2,3}\right)$ de la surface $(S)$ définie par :

$$
\beta=-3 a^{2}-2 a \alpha, \quad \gamma=2 a^{3}+\alpha a^{2}, \quad 0 \leq a<-\frac{\alpha}{3} .
$$

Les rayons de ces cercles sont notés dans ce cas $r_{1}(\alpha, \beta, \gamma)$ et $r_{2,3}(\alpha, \beta, \gamma)$ avec $r_{2,3}(\alpha, \beta, \gamma)<r_{1}(\alpha, \beta, \gamma)$. Le cercle de rayon $r_{1}(\alpha, \beta, \gamma)$ est attractif; celui de rayon $r_{2,3}(\alpha, \beta, \gamma)$ est attractif de l'intérieur, répulsif de l'extérieur. On a en particulier $r_{2,3}(\alpha, \beta, \gamma)=a$.

ii) $(\alpha, \beta, \gamma)$ est dans la partie $\left(S_{1,2}\right)$ de la surface (S) définie par :

$\left(S_{1,2}\right): \quad \beta=-3 a^{2}-2 a \alpha, \quad \gamma=2 a^{3}+\alpha a^{2}, \quad 0<-\frac{\alpha}{3}<a<-\frac{\alpha}{2}$.

Les rayons de ces cercles sont notés dans ce cas $r_{1,2}(\alpha, \beta, \gamma)$ et $r_{3}(\alpha, \beta, \gamma)$ avec $r_{1,2}(\alpha, \beta, \gamma)>r_{3}(\alpha, \beta, \gamma)$. Le cercle de rayon $r_{3}(\alpha, \beta, \gamma)$ est attractif; celui de rayon $r_{1,2}(\alpha, \beta, \gamma)$ est attractif de l'extérieur, répulsif de l'intérieur. On a en particulier $r_{1,2}(\alpha, \beta, \gamma)=a$.

- Il y a trois cycles limites qui sont des cercles centrés à l'origine de rayons respectifs $r_{1}(\alpha, \beta, \gamma)>r_{2}(\alpha, \beta, \gamma)>r_{3}(\alpha, \beta, \gamma)$; ceux de rayons $r_{1}(\alpha, \beta, \gamma)$ et $r_{3}(\alpha, \beta, \gamma)$ sont attractif et celui de rayon $r_{2}(\alpha, \beta, \gamma)$ est répulsif. L'origine est dans ce cas répulsive.

Notons que si $\left(\alpha_{1}, \beta_{1}, \gamma_{1}\right) \in\left(S_{2,3}\right)$ et $\left(\alpha_{2}, \beta_{2}, \gamma_{2}\right) \in\left(S_{1,2}\right)$, alors :

$$
\begin{aligned}
\lim _{\substack{(\alpha, \beta, \gamma) \rightarrow\left(\alpha_{1}, \beta_{1}, \gamma_{1}\right) \\
(\alpha, \beta, \gamma) \in R_{3}}} r_{2}(\alpha, \beta, \gamma) & =\lim _{\substack{\alpha, \beta, \gamma) \rightarrow\left(\alpha_{1}, \beta_{1}, \gamma_{1}\right) \\
(\alpha, \beta, \gamma) \in R_{3}}} r_{3}(\alpha, \beta, \gamma) \\
& =r_{2,3}\left(\alpha_{1}, \beta_{1}, \gamma_{1}\right) \\
- & 321-
\end{aligned}
$$


et

$$
\begin{aligned}
\lim _{\substack{(\alpha, \beta, \gamma) \rightarrow\left(\alpha_{2}, \beta_{2}, \gamma_{2}\right) \\
(\alpha, \beta, \gamma) \in R_{3}}} r_{1}(\alpha, \beta, \gamma) & =\lim _{\substack{(\alpha, \beta, \gamma) \rightarrow\left(\alpha_{2}, \beta_{2}, \gamma_{2}\right) \\
(\alpha, \beta, \gamma) \in R_{3}}} r_{2}(\alpha, \beta, \gamma) \\
& =r_{1,2}\left(\alpha_{2}, \beta_{2}, \gamma_{2}\right) .
\end{aligned}
$$

Remarquons enfin que si $(\alpha, \beta, \gamma)$ est sur la ligne $\left(\ell_{3}\right)$ de $(S)$ définie par $\left(\ell_{3}\right): \quad \alpha=-3 a, \quad \beta=3 a^{2}-2 a \alpha, \quad \gamma=-a^{3}+\alpha a^{2}, \quad 0<a$

alors, il apparait dans le plan des phases un seul cycle limite attractif qui est un cercle centré à l'origine dont le rayon est noté $r_{1,2,3}(\alpha, \beta, \gamma)$ ou encore $r_{1,2,3}(\beta)$; c'est en fait un cercle triple tel que si $\left(\alpha_{0}, \beta_{0}, \gamma_{0}\right) \in\left(\ell_{3}\right)$; alors :

$$
\begin{aligned}
\lim _{\substack{(\alpha, \beta, \gamma) \rightarrow\left(\alpha_{0}, \beta_{0}, \gamma_{0}\right) \\
(\alpha, \beta, \gamma) \in R_{3}}} r_{1}(\alpha, \beta, \gamma) & =\lim _{\substack{\alpha, \beta, \gamma) \rightarrow\left(\alpha_{0}, \beta_{0}, \gamma_{0}\right) \\
(\alpha, \beta, \gamma) \in R_{3}}} r_{2}(\alpha, \beta, \gamma) \\
& =\lim _{\substack{(\alpha, \beta, \gamma) \rightarrow\left(\alpha_{0}, \beta_{0}, \gamma_{0}\right) \\
(\alpha, \beta, \gamma) \in R_{3}}} r_{3}(\alpha, \beta, \gamma) \\
& =r_{1,2,3}\left(\alpha_{0}, \beta_{0}, \gamma_{0}\right) .
\end{aligned}
$$

5.2.2. Diagramme de bifurcation et portraits de phases de $X$

Remarquons d'abord que si on pose

$$
g_{1}\left((\alpha, \beta, \gamma) ; r^{2}\right)=A(\alpha, \beta, \gamma)+B(\alpha, \beta, \gamma) r^{2}+\Gamma(\alpha, \beta, \gamma) r^{4}+O\left(r^{6}\right)
$$

alors la généricité de $X$ implique que l'application qui à $(\alpha, \beta, \gamma)$ associe $(A(\alpha, \beta, \gamma), B(\alpha, \beta, \gamma), \Gamma(\alpha, \beta, \gamma))$ est de rang maximum au point $(0,0,0)$ et donc que modulo un changement de variable dans l'espace des paramètres, on peut supposer que :

$$
g_{1}\left((\alpha, \beta, \gamma) ; r^{2}\right)=\gamma+\beta r^{2}+\alpha r^{4}+O\left(r^{6}\right) .
$$

Par suite, on a :

$$
X=\frac{\partial}{\partial \theta}-G((\alpha, \beta, \gamma) ; \theta, r) \frac{\partial}{\partial r}
$$

avec

$$
G((\alpha, \beta, \gamma) ; \theta, r)=r\left(g_{1}\left((\alpha, \beta, \gamma) ; r^{2}\right)+g_{2}((\alpha, \beta, \gamma) ;(r \cos \theta, r \sin \theta))\right)
$$


Dépendance continue par rapport aux paramètres dans la bifurcation de Hopf-Takens

alors, on a l'inégalité $\left(T_{X}\right)$ suivante :

$$
\frac{\partial}{\partial \gamma}[G((\alpha, \beta, \gamma) ; \theta, r)]<0,
$$

pour tout $(\alpha, \beta, \gamma)$ au voisinage de l'origine des paramètres, $r$ assez petit $>0$ et $\theta \in[0,2 \pi]$.

En outre, d'après $[\mathrm{T}]$ et la généricité de $X$, il est possible de supposer (voir paragraphe 3.2.2) que $X$ et $X^{3,-}$ sont " faiblement identiques " au voisinage de l'origine dans le sens qu'ils ont le même diagramme de bifurcation, les mêmes orbites périodiques et les mêmes singularités sur chaque fibre avec les mêmes types topologiques mais pas nécessairement les mêmes orbites non périodiques.

Soit alors $U=U_{1} \times U_{2}$ un voisinage de l'origine de $\mathbb{R}^{3} \times \mathbb{R}^{2}$ tel que les restrictions de $X$ et de $X^{3,-}$ à $U$ soient faiblement identiques.

Soient $r_{0}>0, \alpha_{0}>0, \beta_{0}>0$ et $\gamma_{0}>0$ tels que :

i) $r_{0}>\sup \left\{r_{i}(\alpha, \beta, \gamma),(i,(\alpha, \beta, \gamma)) \in B \times C\right\}$ où

$$
\begin{aligned}
& B=\{1,2,3,(1,2),(2,3),(1,2,3)\} \\
& C=\left\{(\alpha, \beta, \gamma),-\alpha_{0} \leq \alpha \leq \alpha_{0},-\beta_{0} \leq \beta \leq \beta_{0} \text { et }-\gamma_{0} \leq \gamma \leq \gamma_{0}\right\}
\end{aligned}
$$

ii) $\beta_{0}>\frac{\alpha_{0}^{2}}{3}$ et $\gamma_{0}>\alpha_{0}^{2}+\frac{\alpha_{0}^{3}}{4}$ (fig. 12);

iii) $C \subset U_{1}$;

iv) le disque de l'espace des phases de centre l'origine et de rayon $r_{0}$ soit inclus dans $U_{2}$.

On supposera que l'inégalité $\left(T_{X}\right)$ est vérifiée pour $(\alpha, \beta, \gamma) \in C$ et $\left.r \in] 0, r_{0}\right]$.

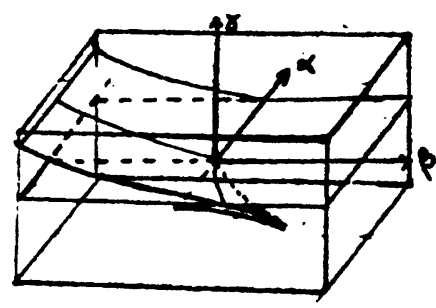

Fig. 12 


\subsubsection{Propriété rotationnelle}

Fixons $\alpha \in\left[-\alpha_{0}, \alpha_{0}\left[, \beta \in\left[-\beta_{0},\left(\inf (\alpha, 0)^{2} / 3\right)[\right.\right.\right.$ et $\left.r \in] 0, r_{0}\right]$ et considérons l'application $\tau$ qui à $\gamma$, tel que $((\alpha, \beta, \gamma), r \cos \theta, r \sin \theta) \in$ $U$, pour $\theta \in[0,2 \pi]$, assecie l'angle $\tau(\gamma)$ de la tangente à l'orbite $D X\left((\alpha, \beta, \gamma) ; \theta_{0}, r\right)$ du champ $X_{(\alpha, \beta, \gamma)}$ au point $\left((\alpha, \beta, \gamma) ; \theta_{0}, r\right)$ de l'axe polaire $\theta=\theta_{0}$ avec ce même axe $\theta=\theta_{0}$. On a :

$$
\operatorname{tg}(\tau(\gamma))=\frac{r}{\dot{r}}=\frac{r}{G((\alpha, \beta, \gamma) ; 0, r)}
$$

Donc $\tau$ est une fonction croissante de $\gamma$ lorsque $(\alpha, \beta, \gamma)$ est voisin de la nappe $(S)$. On a des dessins similaires a ceux de la figure 2.

\subsection{4. Évolution des points fixes sur l'axe des $r$}

Les dessins suivants (fig. 13) décrivent l'évolution de l'axe des $r$ lorsque $(\alpha, \beta, \gamma)$ varie respectivement dans un plan $\left\{\alpha=\alpha_{0}<0\right\}$ et dans le plan $\left\{\alpha=\alpha_{0} \geq 0\right\}$ au voisinage de l'origine de l'espace des paramètres.

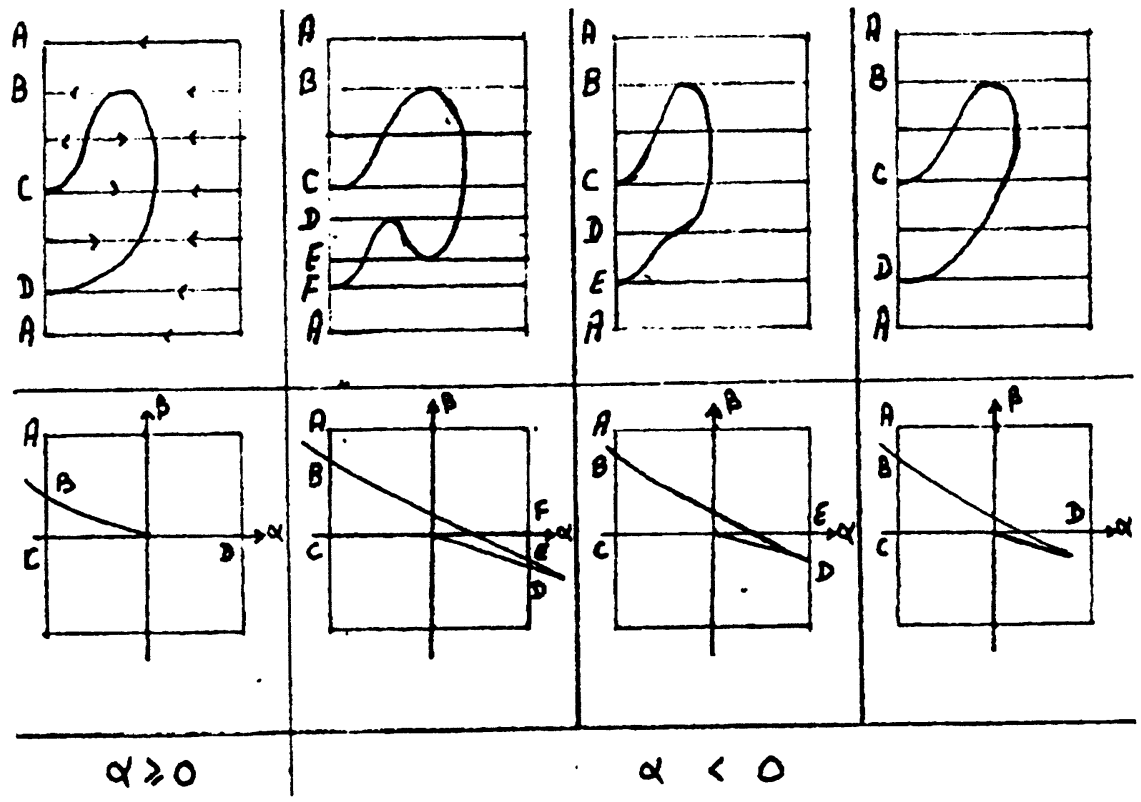

Fig. 13 
Dépendance continue par rapport aux paramètres dans la bifurcation de Hopf-Takens

\subsection{Conjugaison continue entre les difféomorphismes de Poincaré} associés à $X$ et à $X^{3,-}$

Soient $\mathcal{P}$ et $\mathcal{P}^{3,-}$ les applications de Poincaré associées respectivement à $X$ et à $X^{3,-}$ relativement à la demi-droite des $x>0$.

On notera $S_{s}=S_{1} \cup S_{1,2} \cup \ell_{2} \cup \ell_{3}$ et $S_{i}=S_{2,3} \cup \ell_{3} \cup\{(\alpha, 0,0) ; \alpha<0\}$.

\subsubsection{Conjugaison au-dessus de $S_{s}$ et en dessous de $S_{i}$}

On considère $E_{s}$ (resp. $E_{i}$ ) la partie de $C$ au-dessus de $S_{s}$ (resp. en dessous de $S_{i}$ ).

On désigne par $\gamma_{s}$ l'unique réel tel que $\left(\alpha, \beta, \gamma_{s}\right) \in S_{s}$. On note $I=\left[0, r_{0}\right]$ et on définit les intervalles $I(\lambda)=[a(\lambda), b(\lambda)]$ et $I^{\prime}(\lambda)=$ $[c(\lambda), d(\lambda)]$ lorsque $\lambda=(\alpha, \beta, \gamma) \in E_{s}$ par :

$$
\begin{cases}b(\lambda)=r_{0} & \\ a(\lambda)=\frac{1}{2} \inf \left\{r_{1,2}\left(\alpha, \beta, \gamma_{s}\right), \mathcal{P}_{(\alpha, \beta, \gamma)}^{2}\left(r_{0}\right)\right\} & \text { si } \gamma \geq 0 \\ a(\lambda)=\frac{1}{2} \inf \left\{r_{1,2}\left(\alpha, \beta, \gamma_{s}\right)+r_{3}(\alpha, \beta, \gamma), \mathcal{P}_{(\alpha, \beta, \gamma)}^{2}\left(r_{0}\right)\right\} & \text { si } \gamma \leq 0 .\end{cases}
$$

On adopte des définitions analogues pour $c(\lambda)$ et $d(\lambda)$ relativement au champ $X^{3,-}$. On pose :

$$
f(\lambda, r)=\mathcal{P}(\lambda, r), \quad \text { où } \quad \lambda \in E_{s} \text { et } r \in I(\lambda)
$$

et

$$
g(\lambda, r)=\mathcal{P}^{3,-}(\lambda, r), \quad \text { où } \quad \lambda \in E_{s} \text { et } r \in I^{\prime}(\lambda) .
$$

Comme dans le cas Hopf en codimension 2, la propriété rotationnelle de $X$ (resp. $X^{3,-}$ ) par rapport à $\gamma$ au-dessus de $\left(S_{s}\right)$ nous permet d'obtenir à partir du théorème 6 une $\left(C^{0}-C^{0}\right)$-conjugaison de $\mathcal{P}$ vers $\mathcal{P}^{3,-}$ définie pour les valeurs du paramètre appartenant à la région $V_{s}$ de $E_{s}$ comprises entre $\left(S_{s}\right)$ et une surface $\left(\Sigma_{s}\right)$ située au-dessus de $\left(S_{s}\right)$ telle que son intersection avec chaque plan $\alpha=\alpha_{0}$ soit décrite dans la figure 14 .

Il est possible de voir que ce même théorème 6 est applicable lorsqu'on s'intéresse à la region $E_{i} \times I$. On obtient alors une région $V_{i}$ de $E_{i}$ associée à une surface $\left(\Sigma_{i}\right)$ au-dessous de $\left(S_{i}\right)$, voir figure 14 .

Cette $\left(C^{0}-C^{0}\right)$-conjugaison de la restriction de $\mathcal{P}$ à $\left(V_{s} \cup V_{i}\right) \times I$ vers une restriction de $\mathcal{P}^{3,-}$ s'écrit $\phi(\lambda, r)=(\varphi(\lambda), h(\lambda, r))$ et vérifie en particulier 
$h\left(\lambda, r_{0}\right)=r_{0}$ si $\lambda \in\left(V_{s} \cup V_{i}\right), \varphi$ étant un homéomorphisme de $\left(V_{s} \cup V_{i}\right)$ sur son image dans $\left(E_{s} \cup E_{i}\right)$ tel que :

$$
E_{s} \supset \varphi\left(V_{s}\right), \quad E_{i} \supset \varphi\left(V_{i}\right) \text { et } \varphi(\alpha, \beta, \gamma)=\left(\alpha, \beta, \varphi_{3}(\alpha, \beta, \gamma)\right. \text {. }
$$
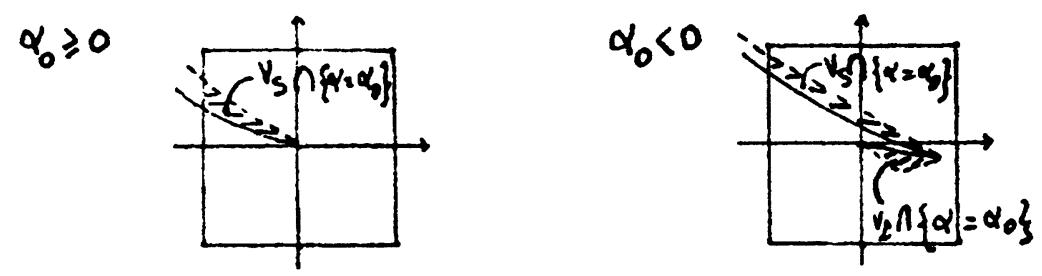

Fig. 14

\subsubsection{Prolongement de la conjugaison à $C \times\left[0, r_{0}\right]$}

Le problème du prolongement de $(\varphi, h)$ à $C \times\left[0, r_{0}\right]$ peut être résolu à l'aide du lemme 2 de prolongement (paragraphe 2.4) d'une façon analogue à celui du cas Hopf en codimension 2 .

On obtient ainsi une $\left(C^{0}-C^{0}\right)$-conjugaison au voisinage de l'origine de $\mathbb{R}^{3} \times \mathbb{R}$ de $\mathcal{P}$ vers $\mathcal{P}^{3,-}$.

5.4. $\left(C^{0}-C^{0}\right)$-conjugaison de la forme normale de $X$ vers $X^{3,-}$

La construction se fait d'une façon similaire à celle faite au paragraphe 3.4.

\section{Remerciements}

Je remercie vivement $\mathrm{F}$. Dumortier pour m'avoir proposé ce travail et pour avoir assuré mon encadrement.

Je remercie J. Sotomayor et R. Roussarie pour l'intérêt qu'ils ont porté à ce travail.

Mes remerciements vont également à la F.N.R.S.T. et à la F.S.T. pour le soutient moral et financier et au L.U.C. pour son acceuil.

\section{Références}

[NPT] Newhouse (S.), Palis (N.) and Takens (F.).- Bifurcations and stability of families of diffeomorphisms,

Publ. Math. I.H.E.S., 57, pp. 5-72. 
Dépendance continue par rapport aux paramètres dans la bifurcation de Hopf-Takens

[PR] Palis (N.) and Roussarie (R.) . - Topological invariants as number of translation,

Lec. Notes in Math., 1125 (1984) pp. 64-86.

[R] RousSARIE (R.) . - Weak and continuous equivalences for families on line diffeomorphisms,

In M.I. Camacho, M.J. Pacifico \& F. Takens. Dynamical Systems and bifurcation theory. Pitman Research Notes in Mathemathics Series 160 (1987) pp. 377-385.

[T] TAKens (F.) .- Unfoldings of certain Singularities of vectorfields, Generalised Hopf bifurcations

J. Diff. Eq. 14 (1973) pp. 476-493.

[Y] Yoccoz (J.CH.) .- Centralisateurs et conjuguaison différentiable des difféomorphismes du cercle,

Thèse, Université d'Orsay (1985). 